\title{
Cramer-Rao Bounds for Deterministic Modal Analysis
}

\author{
Todd McWhorter and Louis L. Scharf, Fellow, IEEE
}

\begin{abstract}
How accurately can deterministic modes be identified from a finite record of noisy data? In this paper we answer this question by computing the Cramer-Rao bound on the error covariance matrix of any unbiased estimator of mode parameters. The bound is computed for many of the standard parametric descriptions of a mode, including autoregressive and moving average parameters, poles and residues, and poles and zeros. Asymptotic, frequency domain versions of the CramerRao bound bring insight into the role played by poles and zeros. Application of the bound to second- and fourth-order systems illustrates the coupling between estimator errors and illuminates the influence of mode locations on our ability to identify them. Application of the bound to the estimation of an energy spectrum illuminates the accuracy of estimators that presume to resolve spectral peaks.
\end{abstract}

\section{INTRODUCTION}

$\mathrm{D}_{\mathrm{s}}$ ETERMINISTIC modal analysis techniques are used to identify the mode parameters $\theta$ in the model

$$
\boldsymbol{y}=\boldsymbol{x}(\boldsymbol{\theta})+\boldsymbol{n}
$$

where $\boldsymbol{y}=\left[\begin{array}{llll}y_{0} & y_{1} & \cdots & y_{N-1}\end{array}\right]^{T}, \boldsymbol{x}(\boldsymbol{\theta})=\left[\begin{array}{lll}x_{0} & x_{1} & \cdots\end{array}\right.$ $\left.x_{N-1}\right]^{T}$, and $n=\left[\begin{array}{llll}n_{0} & n_{1} & \cdots & n_{N-1}\end{array}\right]^{T}$ are the measurement data, modal signal, and noise, respectively. The modal signal $\boldsymbol{x}(\boldsymbol{\theta})$, parameterized by $\boldsymbol{\theta}$, can be modeled as a sum of damped sinusoids (modes), or equivalently, as the deterministic impulse response of an ARMA system of the appropriate order. The ARMA system can, in turn, be parameterized by its AR and MA coefficients, poles and zeros, poles and residues etc. The Cramer-Rao (CR) bound, regardless of the representation utilized, provides an effective measure for evaluating estimators of modal parameters. The purpose of this paper is to derive the CR bounds for this model and to provide general algorithms for their computation.

There is now a comprehensive literature that is addressed to the problem of identifying modes and the parameters that describe them. The literature begins in 1795 with the work of Prony [1] and proceeds to the reducedrank linear prediction techniques of Tufts and Kumaresan [2]-[4]; the least squares (or maximum likelihood) techniques of Evans and Fischl [5], Kumaresan, Scharf, and

Manuscript received June 2, 1991; revised June 22, 1992. The associate editor coordinating the review of this paper and approving it for publication was Prof. Mysore Raghuveer. This work was supported by Bonneville Power Administration under Contract DEB17990BP07346 and by the Office of Naval Research under Contract N00014-89-J-1070.

The authors are with the Department of Electrical and Computer Engineering, University of Colorado, Boulder, CO 80309.

IEEE Log Number 9207531.
Shaw [6], and Bresler and Macovski [7]; the MUSIC methods of Bienvenu and Kopp [8] and Schmidt [9]; and the matrix approximation methods of Kung [10] and Roy and Kailath [11]. But, in spite of the bounds computed in [2], [12]-[14], and the widespread use of the CR bound in the literature, no comprehensive study of performance bounds for deterministic modal analysis, with an ARMA model, has been published.

Our purpose in this paper is to compute Cramer-Rao bounds on the accuracy with which mode parameters may be identified, regardless of the technique used to identify them. The bounds depend only on the information that the data itself carries about the parameters. This information is quantified by the Fisher information matrix. Thus our program is to compute the Fisher information matrix for the AR and MA coefficients of an ARMA $(p, p-1)$ unit pulse response that is observed in additive white Gaussian noise over a finite interval of time. Then, using a transformation formula, we derive the Fisher information matrix for any other equivalent description of the impulse response. When the description is given in terms of poles and residues, then the corresponding parameters are the amplitude, damping coefficient, frequency, and phase for each mode of the unit pulse response. When the observation time is infinite, then the Fisher matrix has a frequency domain interpretation in which spectra and crossspectra play a role. All these formulas may be generalized to account for shaped inputs and colored noise.

In the last few sections of this paper we apply our formulas to second- and fourth-order systems in order to analyze the effect of closely spaced modes on our ability to identify them. We find, for example, that identification errors are correlated so that the high-frequency modulation associated with the average frequency of two closely space modes is well approximated at the expense of poor estimation accuracy for the low-frequency difference (or beat) frequency between the modes.

\section{Statistical Preliminaries}

In this section we intend to provide some background material related to $C R$ bounds and their application to linear models. The first section is used to state the CR bound for a general estimation problem and shows how sensitivity matrices are incorporated in this result. Next we discuss concentration ellipses and their properties and show how they can be used to investigate estimator performance. The remaining sections are used to introduce some 
general forms of the Fisher information matrix when a linear data model is assumed.

\section{A. Cramer-Rao Bounds}

The Cramer-Rao bound is used to lower bound the second order moments of any parametric estimator. A general form of the CR bound can be derived by considering the following estimation problem. Given a data record $y$, we wish to build an estimator $\hat{\boldsymbol{g}}(\boldsymbol{y})$ to estimate the vector function $\gamma=g(\theta)$. Let the Fisher information matrix for the parameter set $\theta$ be $J(\theta)$ and assume that it is invertible. Assume that the estimator $\hat{\mathbf{g}}(\boldsymbol{y})$ is unbiased and denote the estimator covariance matrix as $C_{\hat{g}}=E[(\hat{g}(y)-$ $\left.g(\theta))(\hat{g}(y)-g(\theta))^{T}\right]$. The CR bound for the estimator $\hat{\boldsymbol{g}}(\boldsymbol{y})$ is given by [14]

$$
C_{\hat{g}} \geq T^{T} J^{-1}(\theta) T=J^{-1}(\gamma=g(\theta))
$$

where

$$
T=T(\theta)=\frac{\partial g^{T}(\theta)}{\partial \theta} .
$$

The transformation $\boldsymbol{T}$ merely measures the effects that variations in $\theta$ have on the function $g(\theta)$. Accordingly, we shall refer to matrices of the form in (2.2) as sensitivity matrices. This result states that we can compute the Fisher information matrix for any function of the parameter set $\boldsymbol{\theta}$, from the "base" Fisher information matrix $\boldsymbol{J}(\boldsymbol{\theta})$. In this paper we consider the ARMA parameterization to be the "base" parameter set for modal analysis problems because, as we will show in Sections III, the finite and asymptotic forms of the ARMA Fisher information matrix are computationally tractable and intuitive.

The Fisher information matrix for any equivalent parameter set $\gamma$ can be obtained by letting $\gamma=\boldsymbol{g}(\boldsymbol{\theta})$ where $\boldsymbol{\theta}$ contains the ARMA parameters, and $\boldsymbol{g}(\boldsymbol{\theta})$ is the invertible map from the ARMA coefficients to the alternate parameters. In this instance the sensitivity matrix $T$ is invertible and the Fisher information matrix for the equivalent parameters $\gamma$ is [14]

$$
J(\gamma)=S(\gamma) J\left(\theta=g^{-1}(\gamma)\right) S^{T}(\gamma)
$$

where

$$
S(\gamma)=\frac{\partial\left[g^{-1}(\theta)\right]^{T}}{\partial \gamma}=T^{-1}\left(\theta=g^{-1}(\gamma)\right)
$$

and $g^{-1}(\gamma)$ denotes the inverse map from the paramete1 set $\gamma$ to the ARMA parameters $\theta$. We use (2.3) and (2.4) to compute the Fisher information matrix for the poleresidue and pole-zero parameterizations. Equations (2.1) and (2.2) are used to compute the CR bound for unbiased estimates of the energy spectrum.

\section{B. Error Ellipses}

To provide an introduction to error ellipses, consider a $p$-dimensional unbiased estimator $\theta$ that is normally dis- tributed as $N[\theta, C]$ :

$$
\begin{aligned}
f_{C}(\hat{\boldsymbol{\theta}})= & \frac{1}{(2 \pi)^{p / 2}(\operatorname{det} C)^{1 / 2}} \\
& \cdot \exp \left\{-\frac{1}{2}(\hat{\boldsymbol{\theta}}-\boldsymbol{\theta})^{T} C^{-1}(\hat{\boldsymbol{\theta}}-\boldsymbol{\theta})\right\} .
\end{aligned}
$$

The random variable $r_{C}^{2}=(\hat{\boldsymbol{\theta}}-\boldsymbol{\theta}){ }^{T} \boldsymbol{C}^{-1}(\hat{\boldsymbol{\theta}}-\boldsymbol{\theta})$ is distributed as a chi-squared random variable with $p$ degrees of freedom. The probability that $r_{C}^{2}<r^{2}$ would be determined by integrating the $\chi_{p}^{2}$ density from 0 to $r^{2}$. The volume of Euclidean $p$-space for which $r_{C}^{2} \leq r^{2}$ is defined as the volume of the ellipsoid described by

$$
r_{C}^{2}=(\hat{\boldsymbol{\theta}}-\boldsymbol{\theta})^{T} C^{-1}(\hat{\boldsymbol{\theta}}-\boldsymbol{\theta}) \leq r^{2} .
$$

For $p$ even, the volume of the ellipsoid is

$$
V_{C}=V_{p}(\operatorname{det} C)^{1 / 2} r^{p} ; \quad V_{p}=\frac{(\pi)^{p / 2}}{(p / 2) !}
$$

The diagonal elements of the covariance matrix $\boldsymbol{C}$ are just the variances for the estimators of the parameters $\theta_{i}$. By Hadamard's inequality

$$
\operatorname{det}(\boldsymbol{C}) \leq \prod_{i=1}^{p} c_{i i}=\prod_{i=1}^{p} \operatorname{var}\left(\hat{\boldsymbol{\theta}}_{i}\right)
$$

which implies that for $r=1$

$$
V_{C}^{2} \leq V_{p}^{2} \prod_{i=1}^{p} \operatorname{var}\left(\hat{\boldsymbol{\theta}}_{i}\right) .
$$

This formula indicates that we want the volume of the ellipsoid to be small, since this volume in some sense measures estimator quality. The CR bound for unbiased estimators states that $\boldsymbol{C} \geq \boldsymbol{J}^{-1}$ and this relation implies

$$
V_{J^{-1}}=V_{p}\left(\operatorname{det} J^{-1}\right)^{1 / 2} r^{p} \leq V_{C} .
$$

In other words, the Fisher information matrix for an estimator $\hat{\boldsymbol{\theta}}$ generates the smallest achievable concentration ellipsoid [14]. Not only is the volume $V_{J-1}$ less than the volume $V_{C}$, but the ellipsoid $r_{J^{-1}}=(\hat{\theta}-\theta)^{T} J(\hat{\theta}-\theta) \leq$ $r^{2}$ is entirely contained by the ellipsoid $r_{C}$.

Concentration ellipses can also be used to investigate the interaction of estimator errors. The interaction between any two errors $e_{i}=\left(\hat{\theta}_{i}-\theta_{i}\right)$ and $e_{j}=\left(\hat{\theta}_{j}-\theta_{j}\right)$ may be observed by taking a two-dimensional slice of the concentration ellipsoid as depicted in Fig. 1. The solid ellipse in this figure is the ellipse $r_{C}$ and the dotted ellipse is $r_{J^{-1}}$. The boundaries of the ellipse $r_{C}$ are described by

$$
e^{T} C^{-1} e=1
$$

where $e$ and $C$ are the following $(2 \times 1)$ error vector and $(2 \times 2)$ covariance matrix

$$
\begin{aligned}
\boldsymbol{e} & =\left[\begin{array}{ll}
e_{1} & e_{2}
\end{array}\right]^{T} \\
C & =\left[\begin{array}{ll}
c_{11} & c_{12} \\
c_{12} & c_{22}
\end{array}\right]=\left[\begin{array}{cc}
\sigma_{1}^{2} & \sigma_{1} \sigma_{2} \rho \\
\sigma_{1} \sigma_{2} \rho & \sigma_{2}^{2}
\end{array}\right]
\end{aligned}
$$




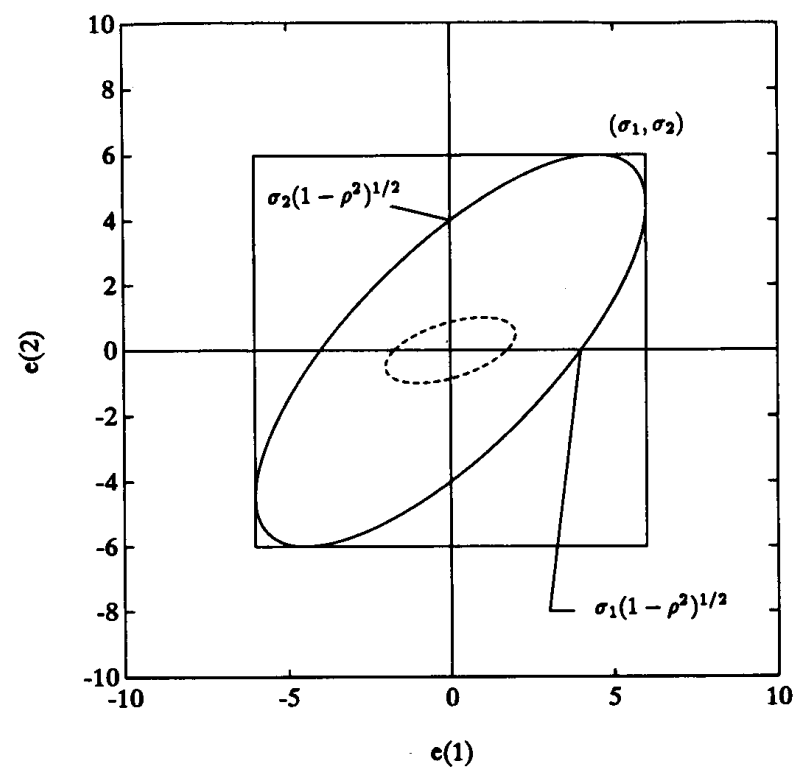

Fig. 1. Concentration ellipses.

and we have let $e_{i}=e_{1}$ and $e_{j}=e_{2}$ for convenience. In the figure, the northeast coordinate of the rectangle that encloses the ellipses is $\left(\sigma_{1}, \sigma_{2}\right)$. These coordinates are just the prior (or marginal) variances of $\hat{\theta}_{1}$ and $\hat{\theta}_{2}$. The intercepts with the $e_{1}$ and $e_{2}$ axes are $\sigma_{1}\left(1-\rho^{2}\right)^{1 / 2}$ and $\sigma_{2}(1$ $\left.-\rho^{2}\right)^{1 / 2}$. These intercepts are just the posterior (or conditional) variances of $\hat{\theta}_{1}$ given $\hat{\theta}_{2}$ and vice versa. If the estimator errors $e_{1}$ and $e_{2}$ are uncorrelated then the axes of the ellipse are just the $e_{1}$ and $e_{2}$ axes. Of course the orientation of the bounding ellipse $r_{J^{-1}}$ may be different than the ellipse $r_{C}$ unless the estimator is efficient. In Section $\mathrm{V}$ we use concentration ellipses to observe the effects of mode spacing on the variance of any efficient estimator of mode parameters. The ellipses exhibit properties which provide additional insight into the interaction of estimation errors. We will defer the discussion of these properties until Section V.

\section{Linear Model}

When the measurements $\boldsymbol{y}=\boldsymbol{x}+\boldsymbol{n}$ consist of a deterministic signal $\boldsymbol{x}$ observed in additive Gaussian noise $\boldsymbol{n}$ : $N[0, \boldsymbol{R}]$, then the Fisher information matrix is [14]

$$
J(\theta)=\left(\frac{\partial x^{T}}{\partial \theta}\right) R^{-i}\left(\frac{\partial x^{T}}{\partial \theta}\right)^{T}
$$

This formula forms the basis for the derivation of the Fisher information matrix for data with structured means. When the signal $\boldsymbol{x}$ obeys the linear, separable model

$$
\boldsymbol{x}=\boldsymbol{H b} ; \quad \boldsymbol{H}=\boldsymbol{H}(\boldsymbol{a})
$$

where $\boldsymbol{a}$ and $\boldsymbol{b}$ are components of the parameter vector $\boldsymbol{\theta}^{T}$ $=\left[b^{T} a^{T}\right]$, then the matrix $\partial x^{T} / \partial \theta$ is

$$
\frac{\partial \boldsymbol{x}^{T}}{\partial \boldsymbol{\theta}}=\left[\begin{array}{l}
\partial \boldsymbol{x}^{T} / \partial \boldsymbol{b} \\
\partial \boldsymbol{x}^{T} / \partial \boldsymbol{a}
\end{array}\right]=\left[\begin{array}{l}
\boldsymbol{H}^{T} \\
\boldsymbol{K}^{T}
\end{array}\right]
$$

$$
\begin{array}{ll}
\boldsymbol{H}=\left[\boldsymbol{h}_{i}\right] ; & \boldsymbol{h}_{i}=\frac{\partial \boldsymbol{x}}{\partial b_{i}} \\
\boldsymbol{K}=\left[\boldsymbol{k}_{i}\right] ; & \boldsymbol{k}_{i}=\frac{\partial \boldsymbol{x}}{\partial a_{i}}=\frac{\partial \boldsymbol{H}}{\partial a_{i}} \boldsymbol{b} .
\end{array}
$$

The Fisher matrix for the linear separable model, assuming $R=\sigma^{2} I$, is [14]

$$
\begin{aligned}
J(b, a) & =\frac{1}{\sigma^{2}}\left[\begin{array}{c}
\boldsymbol{H}^{T} \\
\boldsymbol{K}^{T}
\end{array}\right][\boldsymbol{H} \boldsymbol{K}]=\frac{1}{\sigma^{2}}\left[\begin{array}{ll}
\boldsymbol{H}^{T} \boldsymbol{H} & \boldsymbol{H}^{T} \boldsymbol{K} \\
\boldsymbol{K}^{T} \boldsymbol{H} & \boldsymbol{K}^{T} \boldsymbol{K}
\end{array}\right] \\
& =\frac{1}{\sigma^{2}}\left[\begin{array}{ll}
\left\{\boldsymbol{h}_{i}^{T} \boldsymbol{h}_{j}\right\} & \left\{\boldsymbol{h}_{i}^{T} \boldsymbol{k}_{j}\right\} \\
\left\{\boldsymbol{k}_{i}^{T} \boldsymbol{h}_{j}\right\} & \left\{\boldsymbol{k}_{i}^{T} \boldsymbol{k}_{j}\right\}
\end{array}\right] .
\end{aligned}
$$

In this formula, $\boldsymbol{h}_{i}^{T} \boldsymbol{k}_{j}$ is the $(i, j)$ element of the northeast block, and so on. The matrix inversion lemma for partitioned matrices may be used to invert $J(b, a)$ and obtain

$$
\begin{aligned}
& J^{-1}(\boldsymbol{b}, \boldsymbol{a}) \\
& =\sigma^{2}\left[\begin{array}{cc}
{\left[\boldsymbol{H}^{T}\left(\boldsymbol{I}-\boldsymbol{P}_{K}\right) \boldsymbol{H}\right]^{-1}} & \boldsymbol{F} \\
\boldsymbol{F}^{T} & {\left[\boldsymbol{K}^{T}\left(\boldsymbol{I}-\boldsymbol{P}_{H}\right) \boldsymbol{K}\right]^{-1}}
\end{array}\right]
\end{aligned}
$$

where

$$
\begin{aligned}
\boldsymbol{F} & =-\boldsymbol{H}^{\#} \boldsymbol{K}\left[\boldsymbol{K}^{T}\left(\boldsymbol{I}-\boldsymbol{P}_{H}\right) \boldsymbol{K}\right]^{-1} \\
& =-\left[\boldsymbol{H}^{T}\left(\boldsymbol{I}-\boldsymbol{P}_{K}\right) \boldsymbol{H}\right]^{-1}\left(\boldsymbol{K}^{\#} \boldsymbol{H}\right)^{T}
\end{aligned}
$$

and $\boldsymbol{K}^{\#}$ and $\boldsymbol{H}^{\#}$ are the Moore-Penrose pseudoinverses of $\boldsymbol{K}$ and $\boldsymbol{H}$. The matrices $\boldsymbol{P}_{K}$ and $\boldsymbol{P}_{H}$ are projections and are constructed as $\boldsymbol{P}_{K}=\boldsymbol{K} \boldsymbol{K}^{\#}$ and $\boldsymbol{P}_{H}=\boldsymbol{H} \boldsymbol{H}^{\#}$. This result shows that the error covariance matrix for unbiased estimators of the parameters $\boldsymbol{a}$ and $\boldsymbol{b}$ in the separable linear model is bounded as follows:

$$
\begin{aligned}
\boldsymbol{C}(\boldsymbol{b}) & =E[\hat{\boldsymbol{b}}-\boldsymbol{b}][\boldsymbol{b}-\hat{b}]^{T} \\
& \geq \sigma^{2}\left[\boldsymbol{H}^{T}\left(\boldsymbol{I}-\boldsymbol{P}_{K}\right) \boldsymbol{H}\right]^{-1} \geq \sigma^{2}\left[\boldsymbol{H}^{T} \boldsymbol{H}\right]^{-1} \\
\boldsymbol{C}(\boldsymbol{a}) & =E[\hat{\boldsymbol{a}}-\boldsymbol{a}][\hat{\boldsymbol{a}}-\boldsymbol{a}]^{T} \\
& \geq \sigma^{2}\left[\boldsymbol{K}^{T}\left(\boldsymbol{I}-\boldsymbol{P}_{H}\right) \boldsymbol{K}\right]^{-1} \geq \sigma^{2}\left[\boldsymbol{K}^{T} \boldsymbol{K}\right]^{-1} .
\end{aligned}
$$

The rightmost lower bound $g$ is the CR bound when the subspaces $\langle\boldsymbol{H}\rangle$ and $\langle\boldsymbol{K}\rangle$ are orthogonal. The formulas of (2.19) generate a wealth of geometrical insights into CR bounds. For example, the variance of $\hat{b}_{i}$ depends on the angle that the rank 1 subspace $\left\langle\boldsymbol{h}_{i}\right\rangle$ makes with the subspace $\left\langle\boldsymbol{H} \backslash \boldsymbol{h}_{i}, \boldsymbol{K}\right\rangle$. The geometry of the CR bound is explored in detail in [18].

\section{FISHER INFORMATION MATRIX FOR ARMA PARAMETERIZATION}

In this section we calculate the Fisher information matrix for the ARMA parameterization of the deterministic modal signal in (1.1). The majority of the literature on CR bounds for modal analysis uses the pole-residue characterization for the modal signal. We advocate the ARMA parameterization because the finite and asymptotic forms 
of the Fisher information matrix are computationally tractable and intuitive. In addition, using the transformation formula of Section IV, we can easily obtain the CR bounds for the pole-residue and pole-zero parameterizations.

\section{A. The ARMA Model}

The modal signal $\boldsymbol{x}(\boldsymbol{\theta})$ in the signal-plus-noise model of (1.1) contains the samples $\boldsymbol{x}=\left[\begin{array}{lll}x_{0} & x_{1} \cdots x_{N-1}\end{array}\right]^{T}$. The signal $\left\{x_{t}\right\}$ is generated by forcing the linear system $B(z) / A(z)$ with the unit pulse sequence $\left\{\delta_{t}\right\}$ :

$$
\begin{gathered}
\left\{x_{t}\right\}=\frac{B(z)}{A(z)}\left\{\delta_{t}\right\} \\
\frac{B(z)}{A(z)}=\frac{\sum_{i=0}^{p-1} b_{i} z^{-i}}{\sum_{i=0}^{p} a_{i} z^{-i}} ; \quad\left(a_{0}=1\right) .
\end{gathered}
$$

We have assumed that the degree of $B(z)$ is strictly less than the degree of $A(z)$ to maintain the correspondence between the ARMA model and the pole-residue model. This transfer function model for $\left\{y_{t}\right\}$ may be rewritten in its analysis and synthesis forms:

(analysis) $\quad A(z)\left\{x_{t}\right\}=B(z)\left\{\delta_{t}\right\}=\left\{b_{t}\right\}$

$\left\{b_{t}\right\}=B(z)\left\{\delta_{t}\right\}$ : unit pulse response of MA filter $B(z)$,

(synthesis) $\quad\left\{x_{t}\right\}=B(z)\left\{\frac{1}{A(z)}\left\{\delta_{t}\right\}\right\}=B(z)\left\{h_{t}\right\}$

$\left\{h_{t}\right\}=\frac{1}{A(z)}\left\{\delta_{t}\right\}:$ unit pulse response of AR filter $\frac{1}{A(z)}$.

The AR impulse response $\left\{h_{t}\right\}$ will be fundamental to our study of the Fisher information matrix and we want to emphasize that it is the impulse response of the AR system $1 / A(z)$ and not the ARMA system $B(z) / A(z)$. The sequence $\left\{k_{t}\right\}$ is equally important and is generated by exciting the ARMA system $-B(z) / A(z)$ with the AR sequence $\left\{h_{t}\right\}$.

The analysis and synthesis models for $\left\{x_{t}\right\}$ can be used to generate the equivalent matrix relations:

$$
\begin{aligned}
& \text { (analysis) } \quad G^{-1} x=\left[\begin{array}{l}
b \\
0
\end{array}\right] \text {; } \\
& \boldsymbol{b}=\left[\begin{array}{llll}
b_{0} & b_{1} & \cdots & b_{p-1}
\end{array}\right]^{T} ; \\
& \boldsymbol{a}=\left[\begin{array}{llll}
a_{1} & a_{2} & \cdots & a_{p}
\end{array}\right]^{T} \\
& \boldsymbol{G}^{-1}=\left[\begin{array}{ccccc}
1 & & & & \\
a_{1} & 1 & & & 0 \\
\vdots & & \ddots & & \\
a_{p-1} & \cdots & a_{1} & 1 & \\
\hline & & A^{T} & &
\end{array}\right]
\end{aligned}
$$

$$
\begin{aligned}
& \boldsymbol{A}^{T}=\left[\begin{array}{ccccccc}
a_{p} & \cdots & a_{1} & 1 & & & \\
& a_{p} & \cdots & a_{1} & 1 & & 0 \\
& & \ddots & & \ddots & \ddots & \\
0 & & & a_{p} & \cdots & a_{1} & 1
\end{array}\right] \\
& \text { (synthesis) } \quad x=G\left[\begin{array}{l}
b \\
0
\end{array}\right]=H b
\end{aligned}
$$

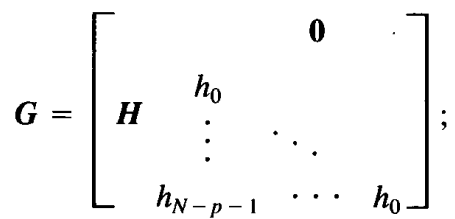

$$
\begin{aligned}
& \boldsymbol{H}=\left[\begin{array}{cccc}
h_{0} & & & \\
h_{1} & h_{0} & & \mathbf{0} \\
\vdots & \ddots & \\
h_{p-1} & \cdots & h_{0} \\
\vdots & & \vdots \\
h_{N-1} & \cdots & h_{N-p}
\end{array}\right]
\end{aligned}
$$

We now have a representation for the modal signal $x$ in terms of the ARMA parameters $\theta^{T}=\left[b^{T} a^{T}\right]$.

\section{B. The Fisher Matrix: Finite and Asymptotic Formulas}

The synthesis model $\boldsymbol{x}=\boldsymbol{H} \boldsymbol{b}$ corresponds to the linear separable model discussed in Section II. Consequently, the Fisher information matrix for the ARMA parameterization has the form established in (2.16), which is reiterated here for convenience:

$$
\begin{aligned}
\boldsymbol{J}(\boldsymbol{b}, \boldsymbol{a})= & \frac{1}{\sigma^{2}}\left[\begin{array}{ll}
\boldsymbol{H}^{T} \boldsymbol{H} & \boldsymbol{H}^{T} \boldsymbol{K} \\
\boldsymbol{K}^{T} \boldsymbol{H} & \boldsymbol{K}^{T} \boldsymbol{K}
\end{array}\right]=\frac{1}{\sigma^{2}}\left[\begin{array}{cc}
\left\{\boldsymbol{h}_{i}^{T} \boldsymbol{h}_{j}\right\} & \left\{\boldsymbol{h}_{i}^{T} \boldsymbol{k}_{j}\right\} \\
\left\{\boldsymbol{k}_{i}^{T} \boldsymbol{h}_{j}\right\} & \left\{\boldsymbol{k}_{i}^{T} \boldsymbol{k}_{j}\right\}
\end{array}\right] \\
= & \frac{1}{\sigma^{2}}\left[\begin{array}{c}
\left\{\boldsymbol{h}_{i}^{T} \boldsymbol{h}_{j}\right\} \\
\left\{\boldsymbol{b}^{T} \frac{\partial \boldsymbol{H}^{T}}{\partial a_{i}} \boldsymbol{h}_{j}\right\} \quad\left\{\boldsymbol{h}_{i}^{T} \frac{\partial \boldsymbol{H}}{\partial a_{j}} \boldsymbol{b}\right\}
\end{array}\right]
\end{aligned}
$$

It is apparent that the vectors $\boldsymbol{k}_{i}$ are $\boldsymbol{k}_{i}=\left(\partial \boldsymbol{H} / \partial a_{i}\right) \boldsymbol{b}$. An alternate expression for the columns $\boldsymbol{k}_{i}$ can be developed that will simultaneously reduce the required computations in forming $J(b, a)$ and provide a foundation for investigating the asymptotic behavior of $J(b, a)$.

To begin, use the synthesis model to express the columns of $\boldsymbol{K}$ as

$$
\boldsymbol{k}_{i}=\frac{\partial \boldsymbol{H}}{\partial a_{i}} \boldsymbol{b}=\frac{\partial \boldsymbol{G}}{\partial a_{i}}\left[\begin{array}{l}
\boldsymbol{b} \\
\mathbf{0}
\end{array}\right] .
$$

Then use the following matrix identity

$$
\frac{\partial G}{\partial a_{i}}=-G \frac{\partial G^{-1}}{\partial a_{i}} \boldsymbol{G}
$$


to obtain [14]

$$
\boldsymbol{k}_{i}=\frac{\partial \boldsymbol{G}}{\partial a_{i}}\left[\begin{array}{l}
\boldsymbol{b} \\
\mathbf{0}
\end{array}\right]=-\boldsymbol{G} \frac{\partial \boldsymbol{G}^{-1}}{\partial a_{i}} \boldsymbol{G}\left[\begin{array}{l}
\boldsymbol{b} \\
\mathbf{0}
\end{array}\right]=-\boldsymbol{G} \frac{\partial \boldsymbol{G}^{-1}}{\partial a_{i}} \boldsymbol{H} \boldsymbol{b} .
$$

The partial derivative $\partial G^{-1} / \partial a_{i}$ is easy to compute, and the resultant matrix is a shift operator of the form

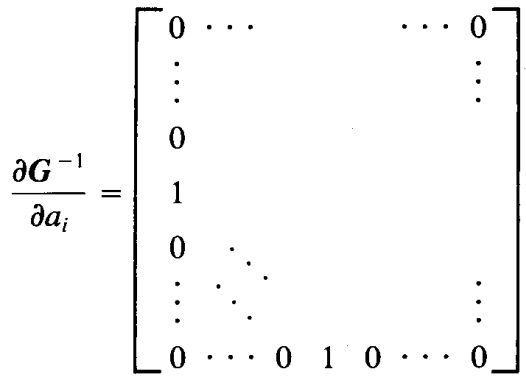

$$
\begin{aligned}
& =Z^{-i} ; \quad Z^{0} \triangleq\left[\begin{array}{cc}
1 & 0 \\
& \\
0 & 1
\end{array}\right]=I .
\end{aligned}
$$

Since the matrix $\boldsymbol{G}$ is a Toeplitz convolution matrix and the corresponding system $1 / A(z)$ is shift invariant, the matrices $G$ and $Z^{-i}$ commute, and (3.11) reduces to
$J_{\infty}(b, a)$ is significant because its inverse represents the "best achievable" bound on the error covariance matrix for unbiased estimates of the parameters, regardless of the duration of the time series.

The asymptotic form for the Fisher information matrix is readily obtained by allowing $N \rightarrow \infty$ in (3.8) and using the representation for $\boldsymbol{h}_{i}$ and $\boldsymbol{k}_{\boldsymbol{i}}$ just derived. The resulting matrix has partitions with $(m, n)$ elements described by

$J_{\infty}(b, a)$

$$
\begin{aligned}
& =\left[\begin{array}{c|c}
\left\langle z^{-(m-1)} \boldsymbol{h}, z^{-(n-1)} \boldsymbol{h}\right\rangle & \left\langle z^{-(m-1)} \boldsymbol{h}, z^{-n} k\right\rangle \\
\hline\left\langle z^{-m} \boldsymbol{k}, \boldsymbol{z}^{-(n-1)} \boldsymbol{h}\right\rangle & \left\langle z^{-m} \boldsymbol{k}, z^{-n} \boldsymbol{k}\right\rangle
\end{array}\right] \\
& =\left[\begin{array}{l|l|l}
\left\langle\boldsymbol{h}, z^{-(n-m)} \boldsymbol{h}\right\rangle & \left\langle\boldsymbol{h}, z^{-(n-m+1)} \boldsymbol{k}\right\rangle \\
\hline\left\langle\boldsymbol{h}, z^{(n-m+1)} \boldsymbol{k}\right\rangle & \left\langle\boldsymbol{k}, z^{-(n-m)} \boldsymbol{k}\right\rangle
\end{array}\right] \\
& =\frac{1}{\boldsymbol{\sigma}^{2}}\left[\frac{\left\{\sum_{i=0}^{\infty} h_{i+n-m} h_{i}\right\}}{\left\{\sum_{i=0}^{\infty} h_{i+n-m+1} k_{i}\right\}}\right] .
\end{aligned}
$$

The terms $\sum_{i} h_{i+n-m} h_{i}$ are deterministic autocorrelations. If it is assumed that the AR system $1 / A(z)$ is stable and causal, then Parseval's rule can be applied to yield a $z$-transform expression for the asymptotic Fisher matrix:

$$
\boldsymbol{J}_{\infty}(\boldsymbol{b}, \boldsymbol{a})=\frac{1}{\sigma^{2}}\left[\frac{\left\{\frac{1}{2 \pi j} \oint H(z) H\left(z^{-1}\right) z^{(n-m)} \frac{d z}{z}\right\} \mid\left\{\frac{1}{2 \pi j} \oint H(z) K\left(z^{-1}\right) z^{(n-m+1)} \frac{d z}{z}\right\}}{\left\{\frac{1}{2 \pi j} \oint H(z) K\left(z^{-1}\right) z^{-(n-m+1)} \frac{d z}{z}\right\} \mid\left\{\frac{1}{2 \pi j} \oint K(z) K\left(z^{-1}\right) z^{(n-m)} \frac{d z}{z}\right\}}\right] .
$$

$$
\begin{aligned}
& \boldsymbol{k}_{\boldsymbol{i}}=-\boldsymbol{Z}^{-i} \boldsymbol{G} \boldsymbol{H} \boldsymbol{b}=\boldsymbol{Z}^{-i} \boldsymbol{k} \\
& =\left[\begin{array}{lllllll}
0 & \cdots & 0 & k_{0} & k_{1} & \cdots & k_{N-1-i}
\end{array}\right]^{T} \text {; } \\
& i=1,2, \cdots, p
\end{aligned}
$$

where $\boldsymbol{k}=\left[k_{0}, k_{1}, \cdots, k_{N-1}\right]^{T}$ contains a snapshot of the signal $\left\{k_{t}\right\}=[-B(z) / A(z)]\left\{h_{t}\right\}$. In order to compute $J(b, a)$, it is only necessary to compute the sequences $\left\{h_{t}\right\}$ and $\left\{k_{t}\right\}$, and form the matrices $\boldsymbol{H}$ and $\boldsymbol{K}$ by appropriate shifts and inner products of $\left\{h_{t}\right\}$ and $\left\{k_{t}\right\}$.

In summary, the Fisher matrix is described by (3.8) with $\boldsymbol{h}_{i} \doteq\left[\begin{array}{llllll}0 & \cdots & 0 & h_{0} & \cdots & h_{N-i}\end{array}\right]^{T}, \quad\left\{h_{t}\right\}=$ $(1 / \boldsymbol{A}(z))\left\{\delta_{t}\right\}$, and $\boldsymbol{k}_{i}=\left[\begin{array}{llllll}0 & \cdots & 0 & k_{0} & \cdots & k_{N-1-i}\end{array}\right]^{T}$, $\left\{k_{t}\right\}=[-B(z) / A(z)]\left\{h_{t}\right\}$ for $i=1,2, \cdots, p$. This completes our characterization of the Fisher matrix $J(b, a)$ for the ARMA model $\left\{x_{t}\right\}=[B(z) / A(z)]\left\{\delta_{t}\right\}$ for finite samples.

It is apparent in the preceding development that the Fisher information matrix $J(b, a)$ is a function of the modal parameters $\boldsymbol{\theta}^{T}=\left[\boldsymbol{b}^{T} \boldsymbol{a}^{T}\right]$ and the duration $N$ of the time series $\left\{x_{t}\right\}$. In the following paragraphs we will derive the asymptotic form of the ARMA Fisher information matrix by assuming that an infinite record of data is available $(N \rightarrow \infty)$. The asymptotic Fisher information matrix
This means that the asymptotic Fisher matrix may be written as

$$
\boldsymbol{J}_{\infty}(\boldsymbol{b}, \boldsymbol{a})=\frac{1}{\sigma^{2}}\left[\begin{array}{ll}
\boldsymbol{R}_{h h} & \boldsymbol{R}_{h k} \\
\boldsymbol{R}_{k h} & \boldsymbol{R}_{k k}
\end{array}\right]
$$

where the correlation matrices $\boldsymbol{R}_{h h}, \boldsymbol{R}_{h k}$, and $\boldsymbol{R}_{k k}$ are composed of the elements $\boldsymbol{R}_{h h}=\left\{r_{h h}(n-m)\right\}, \boldsymbol{R}_{h k}=\left\{r_{h k}(n\right.$ $-m+1)\}$, and $\boldsymbol{R}_{k k}=\left\{r_{k k}(n-m)\right\}$. Fig. 2 illustrates how to construct the sequence $\left\{h_{t}\right\}$ and $\left\{k_{t}\right\}$ that have the deterministic auto- and cross-correlation sequences $r_{h h}(n)$, $r_{h k}(n)$, and $r_{k k}(n)$ that build the Fisher matrix. In this characterization, $\left\{h_{t}\right\}$ is the response of $1 / A(z)$ to an impulse. Similarly, $\left\{k_{t}\right\}$ is the response of $-B(z) / A(z)$ to the sequence $\left\{h_{t}\right\}$.

\section{Algorithm for Generating the Asymptotic Fisher Matrix}

The terms in the northwest and southeast partitions of the asymptotic Fisher information matrix $\boldsymbol{J}_{\infty}(\boldsymbol{b}, \boldsymbol{a})$ are elements of deterministic autocorrelation sequences. Similarly, the terms in the northeast and southwest partitions of $J_{\infty}(b, a)$ are components of deterministic cross-correlation sequences. A technique for computing autocorre- 
$\left\{\delta_{t}\right\}$

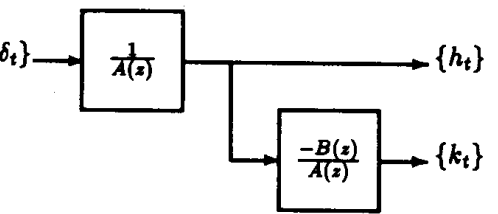

Fig. 2. Sequences associated with the asymptotic Fisher matrix

lation sequences was introduced by Dugré et al. [15]. This method was generalized by Demeure and Mullis [16] to include computation of cross-correlation sequences. In addition, Demeure and Mullis derived fast algorithms for performing the required computations. In the remainder of this section we will summarize some of these techniques within the context of computing $\boldsymbol{J}_{\infty}(\boldsymbol{b}, \boldsymbol{a})$.

Autocorrelation sequences can be considered a special case of the more general class of cross-correlation sequences. However, the algorithm for computing autocorrelation exhibits a symmetry that can be exploited to reduce the complexity of the calculation. Accordingly, we will first present a method for computing the autocorrelations that build the diagonal partitions of $\boldsymbol{J}_{\infty}(\boldsymbol{b}, \boldsymbol{a})$. The terms in the diagonal partitions of the asymptotic Fisher matrix have the general form

$$
r_{k}=\frac{1}{2 \pi j} \oint \frac{F(z)}{G(z)} \frac{F\left(z^{-1}\right)}{G\left(z^{-1}\right)} z^{k} \frac{d z}{z}
$$

where $F(z)=1, G(z)=A(z)$ for the northwest partition and $F(z)=-B(z), G(z)=A^{2}(z)$ for the southeast partition. From this equation it is apparent that the sequence $\left\{r_{k}\right\}$ can be determined by

$$
\left\{r_{k}\right\}=F(z) F\left(z^{-1}\right) / G(z) G\left(z^{-1}\right)\left\{\delta_{k}\right\} .
$$

Since we have constrained the roots of $A(z)$ to lie inside the unit circle, the system $F(z) F\left(z^{-1}\right) / G(z) G\left(z^{-1}\right)$ can be easily factored into its causal part $Y(z) / G(z)$ and anticausal part $Y\left(z^{-1}\right) / G\left(z^{-1}\right)$. That is,

$$
\frac{F(z) F\left(z^{-1}\right)}{G(z) G\left(z^{-1}\right)}=\frac{Y(z)}{G(z)}+\frac{Y\left(z^{-1}\right)}{G\left(z^{-1}\right)}
$$

or

$$
F(z) F\left(z^{-1}\right)=Y(z) G\left(z^{-1}\right)+Y\left(z^{-1}\right) G(z)
$$

which is a special case of Euclid's equation. To generate $\left\{r_{k}\right\}$ we need only to determine $Y(z)$ and compute the elements of the impulse response of the system $Y(z) / G(z)$, since the correlation sequence $\left\{r_{k}\right\}$ is symmetric. To determine $Y(z)$, the first step is to compute the product $F(z) F\left(z^{-1}\right)$ and decompose this square into its causal and anticausal pieces:

$$
\begin{gathered}
F(z) F\left(z^{-1}\right)=C(z)+C\left(z^{-1}\right) ; \quad F(z)=\sum_{i=0}^{m} f_{i} z^{-i} \\
C(z)=\sum_{i=0}^{m} c_{i} z^{-i}
\end{gathered}
$$

From equation (3.19) we can derive the following expression for the coefficients of $C(z)$ :

$$
c_{i}= \begin{cases}\frac{1}{2} \sum_{k=0}^{m} f_{k}^{2}, & i=0 \\ \sum_{k=0}^{m} f_{k} f_{k+i}, & 1 \leq i \leq m .\end{cases}
$$

Recall that $f_{k}=\delta_{k}$ for $F(z)=1$ and $f_{k}=-b_{k}$ for $F(z)=$ $-B(z)$. Equation (3.18) can now be written as

$$
\begin{gathered}
Y(z) G\left(z^{-1}\right)+Y\left(z^{-1}\right) G(z)=C(z)+C\left(z^{-1}\right) ; \\
G(z)=\sum_{i=0}^{m} g_{i} z^{-i} .
\end{gathered}
$$

This is Jury's equation which must be solved for the polynomial $Y(z)$. Existence of the solution is guaranteed if $G(z)$ is minimum phase. In our case the condition is satisfied since we stipulated that $A(z)$ is minimum phase. Equation (3.21) can be expressed in matrix notation as

$$
\begin{gathered}
{\left[\begin{array}{ccccc}
g_{0} & g_{1} & g_{2} & \cdots & g_{m} \\
g_{1} & g_{0}+g_{2} & g_{1}+g_{3} & \cdots & g_{m-1} \\
g_{2} & g_{3} & g_{0}+g_{4} & \cdots & g_{m-2} \\
\vdots & \vdots & \vdots & & \vdots \\
g_{m} & 0 & 0 & \cdots & g_{0}
\end{array}\right]\left[\begin{array}{c}
y_{0} \\
y_{1} \\
\vdots \\
y_{m}
\end{array}\right]} \\
=\left[\begin{array}{c}
c_{0} \\
c_{1} \\
\vdots \\
c_{m}
\end{array}\right] \\
\boldsymbol{M}^{\top} \boldsymbol{y}=\boldsymbol{c}
\end{gathered}
$$

where the matrix $G$ is

$$
\boldsymbol{M}^{T}=\left[\left\{\begin{array}{cl}
g_{j-1:} & i=1 \\
g_{i+j-2}+g_{j-i} ; & i \neq 1
\end{array}\right\}_{i, j}\right] .
$$

The matrix $\boldsymbol{M}^{T}$ is the transpose of the Jury matrix defined in [15] and [16]. The coefficients of $Y(z)$ can be obtained by solving the linear set of equations in (3.22) using any standard technique. A fast algorithm for solving (3.22) can be found in [16]. Let $\left\{\alpha_{t}\right\}$ denote the impulse response of the system $Y(z) / G(z)$ where $Y(z)$ is the polynomial formed from $y$ of (3.22). Then the diagonal partitions of $\boldsymbol{J}_{\infty}(\boldsymbol{b}, \boldsymbol{a})$ are Toeplitz correlation matrices with elements

$$
r_{t}= \begin{cases}2 \alpha_{0}, & t=0 \\ \alpha_{t}, & 1 \leq t \leq p-1 .\end{cases}
$$

Note that we need to perform the algorithm for each partition individually. 
The terms in the northeast and southwest partitions of the asymptotic Fisher information matrix have the form

$$
r_{k}=\frac{1}{2 \pi j} \oint \frac{1}{A(z)} K\left(z^{-1}\right) z^{k} \frac{d z}{z} ; \quad K\left(z^{-1}\right)=-\frac{B\left(z^{-1}\right)}{A^{2}\left(z^{-1}\right)}
$$

Accordingly, the deterministic cross-correlation sequence $\left\{r_{t}\right\}$ can be determined by $A(z)\left\{r_{i}\right\}=K\left(z^{-1}\right)\left\{\delta_{t}\right\}$. This sequence does not exhibit the symmetry of the deterministic autocorrelation sequence, and the sign of the index $k$ is significant. Nonetheless, the approach of the previous paragraphs can be modified to compute $\left\{r_{t}\right\}$ using the techniques of [16] and [17].

\section{Sensitivity Matrices for Alternate PARAMETERIZATIONS}

The Fisher information matrices for the pole-residue and pole-zero parameterizations can be derived from $\boldsymbol{J}(\boldsymbol{b}, \boldsymbol{a})$ by calculating the sensitivity matrix for each representation and using (2.6). This method is particularly useful for obtaining the asymptotic forms of the Fisher information matrices for these two parameterizations. In this section we derive explicit formulas for the sensitivity matrices corresponding to the pole-residue and pole-zero parameterizations. We also derive a sensitivity matrix of the form specified in (2.3) that enables us to compute the CR bound for unbiased estimators of the energy spectrum for a deterministic modal signal.

\section{A. Sensitivity Matrix for Pole-Residue Parameterization}

The pole-residue representation for the modal signal $\left\{x_{1}\right\}$ is

$$
\begin{gathered}
\left\{x_{i}\right\}=\sum_{i=1}^{p} c_{i}\left|\frac{1}{1-z_{i} z^{-1}}\left\{\delta_{i}\right\}\right|=\sum_{i=1}^{\prime} c_{i} z_{i}^{\prime} . \\
\text { (residue) } c_{i}=\frac{A_{i}}{2} e^{j \phi_{i}} ; \quad \text { (pole) } z_{i}=\rho_{i} e^{j \theta_{i}}
\end{gathered}
$$

We have assumed that the time series $\left\{x_{l}\right\}$ has only real valued elements. Consequently, the poles and residues appear in conjugate pairs. The multiplicity of the poles is not an issue in the computation of $\boldsymbol{J}(\boldsymbol{b}, \boldsymbol{a})$, however pole multiplicity does affect the form of the sensitivity matrix. In the computations that follow, we have assumed that all poles are simple; that is, $z_{i} \neq z_{j}$, for $i \neq j$. If the model order $p=2 q$. is even and the constraints just enumerated are enforced, then

$$
x_{i}=\sum_{i=1}^{q} A_{i} \rho_{i}^{t} \cos \left(\theta_{i} t+\phi_{i}\right)
$$

is a linear combination of damped, weighted, and phased sinusoids. The relationship between the pole-residue and ARMA representations can be expressed in the frequency domain as

$$
\begin{aligned}
A(z) & =1+\sum_{i=1}^{p} a_{i} z^{-i} \\
& =\prod_{n=1}^{q}\left(1-2 \rho_{n} \cos \left(\theta_{n}\right) z^{-1}+\rho_{n}^{2} z^{-2}\right) \\
\frac{B(z)}{A(z)} & =\frac{\sum_{i=0}^{p-1} b_{i} z^{-i}}{1+\sum_{i=1}^{p} a_{i} z^{-i}} \\
& =\sum_{n=1}^{q} \frac{\left(A_{n} \cos \left(\phi_{n}\right)-A_{n} \rho_{n} \cos \left(\theta_{n}-\phi_{n}\right) z^{-1}\right)}{\left(1-2 \rho_{n} \cos \left(\theta_{n}\right) z^{-1}+\rho_{n}^{2} z^{-2}\right)} .
\end{aligned}
$$

These equations summarize the transformation $\theta=$ $\boldsymbol{g}^{-1}(\gamma)$, where $\boldsymbol{\theta}^{T}=\left[\begin{array}{ll}\boldsymbol{b}^{T} & \boldsymbol{a}^{T}\end{array}\right]$ and $\boldsymbol{\gamma}^{T}=\left[\begin{array}{llll}\boldsymbol{A}^{T} & \phi^{T} & \boldsymbol{\rho}^{T} & \boldsymbol{\theta}^{T}\end{array}\right]$. If $p$ is odd, (4.2) and (4.3) can be easily extended to include a pole and residue with $\theta_{i}=0$ and $\phi_{i}=0$, respectively.

The $(2 p \times 2 p)$ sensitivity matrix for the transformation $\boldsymbol{\theta}=g^{-1}(\gamma)$ is

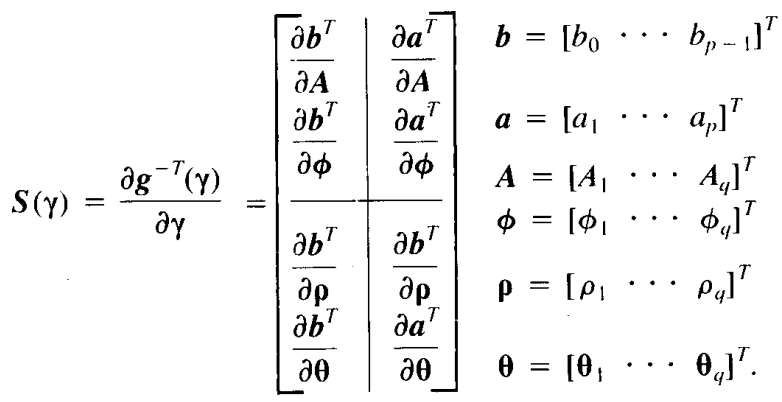

As described in Section II, this sensitivity matrix, in conjunction with the Fisher information matrix for the ARMA representation, can be used to generate the Fisher information matrix for the pole-residue characterization:

$$
J(\gamma)=S(\gamma) J\left(\theta=g^{-1}(\gamma)\right) S^{T}(\gamma) .
$$

It is apparent from the transformation $\theta=g^{-1}(\gamma)$ that the northeast partition of $S(\gamma)$ is zero:

$$
\left[\begin{array}{l}
\frac{\partial a^{T}}{\partial A} \\
\frac{\partial a^{T}}{\partial \phi}
\end{array}\right]=0
$$

We can express the relationship between the AR parameters and the pole parameters $\left(\rho_{i}, \boldsymbol{\theta}_{i}\right)$ in matrix form

$$
L=C_{1} \quad C_{2} \cdots C_{q}
$$


where $q=p / 2$ and

$$
\begin{aligned}
& L=\left[\begin{array}{ccccc}
1 & a_{1} & a_{2} & \cdots & a_{p} \\
& 1 & a_{1} & \cdots & a_{p-1} \\
& & & \vdots \\
& 0 & & & 1
\end{array}\right]=\left[\begin{array}{cc}
1 & a^{T} \\
0 & \tilde{L}
\end{array}\right]
\end{aligned}
$$

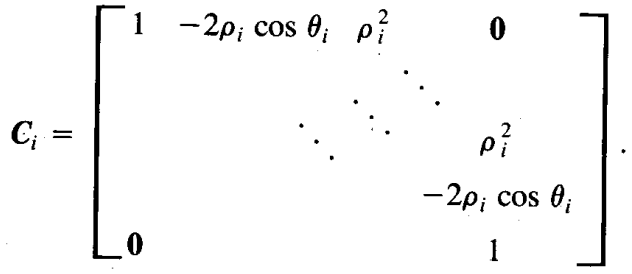

The matrix products on the right-hand side of (4.7) are performing convolution. Since convolution is commutative for linear systems, we can permute the matrix factors in (4.7) and preserve the equality. The matrix $C_{i}$ is the only factor which is a function of $\rho_{i}$ and we can use the commutativity property to obtain

$$
\frac{\partial \boldsymbol{L}}{\partial \rho_{i}}=\frac{\partial \boldsymbol{C}_{i}}{\partial \rho_{i}} \boldsymbol{C}_{i}^{-1} \boldsymbol{L}
$$

The matrix $C_{i}$ is a convolution matrix for the FIR system $A_{i}(z)=1-2 \rho_{i} \cos \theta_{i} z^{-1}+\rho_{i}^{2} z^{-2}$. This implies that the matrix $C_{i}$ is composed of terms from the impulse response sequence for the AR system $1 / A_{i}(z)$. The $k$ th term of this sequence, denoted as $h_{i}(k)$, is given by $h_{i}(k)=\rho_{i}^{k} \sin$ $\left(\theta_{i}(k+1)\right) / \sin \theta_{i}$. If $\theta_{i}=0$, the poles are real and the matrix factor $C_{i}$ will not correspond to the form shown previously. The equations that follow must be modified to account for this difference. If $\theta_{i} \neq 0$, the matrix $\partial L / \partial \rho_{i}$ has the form

$$
\frac{\partial L}{\partial \rho_{i}}=\left[\begin{array}{ll}
\mathbf{0} & \alpha_{i} \\
0 & \mathbf{0}^{T}
\end{array}\right] L
$$

where the matrix $\alpha_{i}$ is

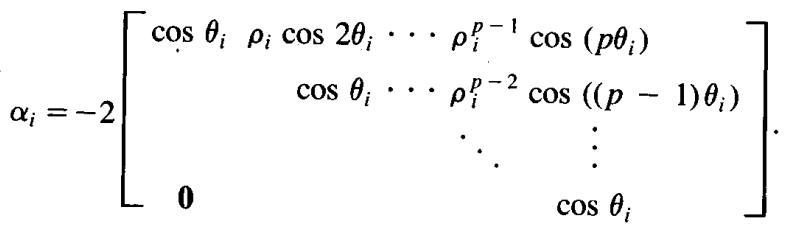

A similar set of calculations can be performed for the parameter $\theta_{i}$ to obtain

$$
\frac{\partial L}{\partial \theta_{i}}=\left[\begin{array}{ll}
\mathbf{0} & \beta_{i} \\
0 & \mathbf{0}^{T}
\end{array}\right] L,
$$

$$
\beta_{i}=2\left[\begin{array}{cccc}
\rho_{i} \sin \theta_{i} & \rho_{i}^{2} \sin 2 \theta_{i} \cdots & \rho_{i}^{p} \sin \left(p \theta_{i}\right) \\
& \rho_{i} \sin \theta_{i} \cdots \rho_{i}^{p-1} & \sin \left((p-1) \theta_{i}\right) \\
& \ddots & \vdots \\
0 & & \ddots & \rho_{i} \sin \theta_{i}
\end{array}\right] .
$$

Note, from (4.8), that the first row of the matrix $L$ contains the vector $a^{T}$. We can use this fact, in conjunction with (4.11) and (4.13), to generate the matrices in the southeast partition of $S(\gamma)$

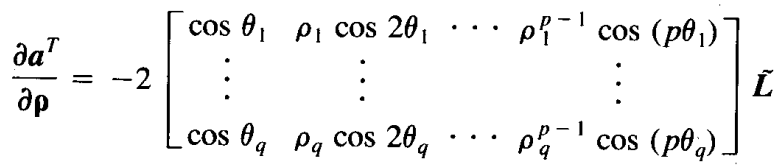

$$
\begin{aligned}
& \frac{\partial \boldsymbol{a}^{T}}{\partial \boldsymbol{\theta}}=2\left[\begin{array}{cccc}
\rho_{1} \sin \theta_{1} & \rho_{1}^{2} \sin 2 \theta_{1} & \cdots & \rho_{1}^{p} \sin \left(p \theta_{1}\right) \\
\vdots & \vdots & & \vdots \\
\rho_{q} \sin \theta_{q} & \rho_{q}^{2} \sin 2 \theta_{q} & \cdots & \rho_{q}^{p} \sin \left(p \theta_{q}\right)
\end{array}\right] \tilde{\boldsymbol{L}} \\
& \tilde{\boldsymbol{L}}=\left[\begin{array}{cccc}
1 & a_{1} & \cdots & a_{p-1} \\
& 1 & \cdots & a_{p-2} \\
& & & \vdots \\
0 & & & 1
\end{array}\right] .
\end{aligned}
$$

The matrices in the remaining partitions of $S(\gamma)$ can be computed from the relation

$$
\boldsymbol{b}^{T}=\boldsymbol{A}^{T} \boldsymbol{V}^{T} \tilde{\boldsymbol{L}}
$$

where $\boldsymbol{A}$ and $\tilde{\boldsymbol{L}}$ have already been defined and $\boldsymbol{V}^{T}$ is the following row-Vandermonde matrix:

$$
\begin{aligned}
\boldsymbol{V}^{T}= & {\left[\begin{array}{cc}
\cos \phi_{1} & \rho_{1} \cos \left(\theta_{1}+\phi_{1}\right) \cdots \\
\vdots & \vdots \\
\cos \phi_{q} & \rho_{q} \cos \left(\theta_{q}+\phi_{q}\right) \cdots \\
& \cdots \rho_{1}^{p-1} \cos \left((p-1) \theta_{1}+\phi_{1}\right) \\
& \cdots \\
& \cdots \rho_{q}^{p-1} \cos \left((p-1) \theta_{q}+\phi_{q}\right)
\end{array}\right] }
\end{aligned}
$$

This relation is generated by equating the ARMA representation for $\boldsymbol{x}$ with the pole-residue characterization for $x$, over time $t=0,1, \cdots, p-1$. From (4.18) it is obvious that

$$
\frac{\partial \boldsymbol{b}^{T}}{\partial \boldsymbol{A}}=\boldsymbol{V}^{T} \tilde{\boldsymbol{L}}
$$

Since the AR parameters are independent of the mode 
phase, we can also obtain

$\frac{\partial b^{T}}{\partial \phi_{i}}=A^{T} \frac{\partial V^{T}}{\partial \phi_{i}} \tilde{L}$

$$
\begin{aligned}
\frac{\partial \boldsymbol{b}^{T}}{\partial \boldsymbol{\phi}}=- & {\left[\begin{array}{cc}
A_{1} \sin \phi_{1} & A_{1} \rho_{1} \sin \left(\theta_{1}+\phi_{1}\right) \cdots \\
\vdots & \vdots \\
A_{q} \sin \phi_{q} & A_{q} \rho_{q} \sin \left(\theta_{q}+\phi_{q}\right) \cdots \\
& \cdots A_{1} \rho_{1}^{p-1} \sin \left((p-1) \theta_{1}+\phi_{1}\right) \\
& \cdots A_{q} \rho_{q}^{p-1} \sin \left((p-1) \theta_{q}+\phi_{q}\right)
\end{array}\right] \tilde{L} . }
\end{aligned}
$$

The AR parameters are a function of the mode parameters $\rho_{i}$ and $\theta_{i}$. This implies that we need to know the forms of $\partial \tilde{L} / \partial \rho_{i}$ and $\partial \tilde{L} / \partial \theta_{i}$. These can be obtained from (4.11) and (4.13) and are stated below:

$$
\frac{\partial \tilde{L}}{\partial \rho_{i}}=\left[\begin{array}{ll}
\mathbf{0} & \tilde{\alpha}_{i} \\
0 & \mathbf{0}^{T}
\end{array}\right] \tilde{L}=\tilde{\boldsymbol{X}}_{i} \tilde{L}
$$

$\tilde{\alpha}_{i}=$

$=-2\left[\begin{array}{cccc}\cos \theta_{i} & \rho_{i} \cos 2 \theta_{i} & \cdots & \rho_{i}^{p-2} \cos \left((p-1) \theta_{i}\right) \\ & \cos \theta_{i} & \cdots & \rho_{i}^{p-2} \cos \left((p-2) \theta_{i}\right) \\ & & \ddots & \vdots \\ 0 & & & \cos \theta_{i}\end{array}\right]$

$$
\frac{\partial \tilde{L}}{\partial \theta_{i}}=\left[\begin{array}{ll}
\mathbf{0} & \tilde{\beta}_{i} \\
0 & \mathbf{0}^{T}
\end{array}\right] \tilde{L}=\tilde{Y}_{i} \tilde{L}
$$

$\tilde{\beta}_{i}=$

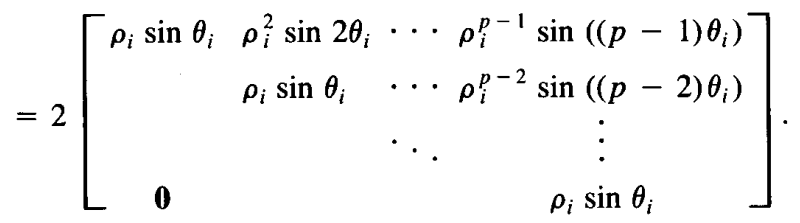

The chain rule can be used to derive

$$
\begin{aligned}
& \frac{\partial \boldsymbol{b}^{T}}{\partial \rho_{i}}=\boldsymbol{A}^{T}\left[\frac{\partial \boldsymbol{V}^{T}}{\partial \rho_{i}}+\boldsymbol{V}^{T} \tilde{\boldsymbol{X}}_{i}\right] \tilde{\boldsymbol{L}} \\
& \frac{\partial \boldsymbol{b}^{T}}{\partial \theta_{i}}=\boldsymbol{A}^{T}\left[\frac{\partial \boldsymbol{V}^{T}}{\partial \theta_{i}}+\boldsymbol{V}^{T} \tilde{\boldsymbol{Y}}_{i}\right] \tilde{\boldsymbol{L}} .
\end{aligned}
$$

Equations (4.27) and (4.28) characterize the $i$ th rows of $\partial b^{T} / \partial \rho$ and $\partial b^{T} / \partial \theta$, respectively.

\section{B. Sensitivity Matrix for Pole-Zero Parameterization}

The pole-zero representation for the modal signal is

$$
\left\{x_{t}\right\}=\frac{b_{0} \prod_{n=1}^{p-1}\left(1-w_{n} z^{-1}\right)}{\prod_{n=1}^{p}\left(1-z_{n} z^{-1}\right)}\left\{\delta_{t}\right\}
$$

where $b_{0}, w_{n}$, and $z_{n}$ correspond to the gain, zeros, and poles, respectively. As the time series $\left\{x_{t}\right\}$ is constrained to be strictly real, then the poles $z_{i}=\rho_{i} e^{j \theta_{i}}$ must appear in conjugate pairs. The same restriction also applies to the zeros $w_{i}=r_{i} e^{j \phi_{i}}$. Additionally, we stipulate that

$$
\begin{array}{rl}
z_{i} \neq z_{j} & i \neq j \\
z_{i} \neq w_{j} & \text { for all } i \text { and } j \\
w_{i} \neq w_{j} & i \neq j .
\end{array}
$$

That is, all poles and zeros are simple and no pole-zero cancellation is permitted. If, in a particular application, multiple poles or zeros are present in the system model, the sensitivity matrix for the parameters can be derived, but not by using the techniques described in the following paragraphs. Again we will assume that $p=2 q$ is even. The relationship between the pole-zero parameterization and the ARMA representation is given by

$$
\begin{aligned}
B(z)= & \sum_{i=0}^{p-1} b_{i} z^{-i}=b_{0}\left(1-r_{0} z^{-1}\right) \prod_{n=1}^{q-1} \\
& \cdot\left(1-2 r_{n} \cos \left(\phi_{n}\right) z^{-1}+r_{n}^{2} z^{-2}\right) \\
A(z)= & 1+\sum_{i=1}^{p} a_{i} z^{-i}=\prod_{n=1}^{q} \\
& \cdot\left(1-2 \rho_{n} \cos \left(\theta_{n}\right) z^{-1}+\rho_{n}^{2} z^{-2}\right) .
\end{aligned}
$$

These equations summarize the transformation $\theta=$ $\boldsymbol{g}^{-1}(\gamma)$, where $\boldsymbol{\theta}^{T}=\left[\begin{array}{ll}\boldsymbol{b}^{T} & \boldsymbol{a}^{T}\end{array}\right]$ and $\boldsymbol{\gamma}^{T}=$ $\left[\begin{array}{lllll}b_{0} & \boldsymbol{r}^{T} & \boldsymbol{\phi}^{T} & \boldsymbol{\rho}^{T} & \boldsymbol{\theta}^{T}\end{array}\right]$. As in the pole-residue characterization, these equations can be easily modified if the model order $p$ is odd.

The sensitivity matrix for the transformation from the ARMA to the pole-zero characterization is given by

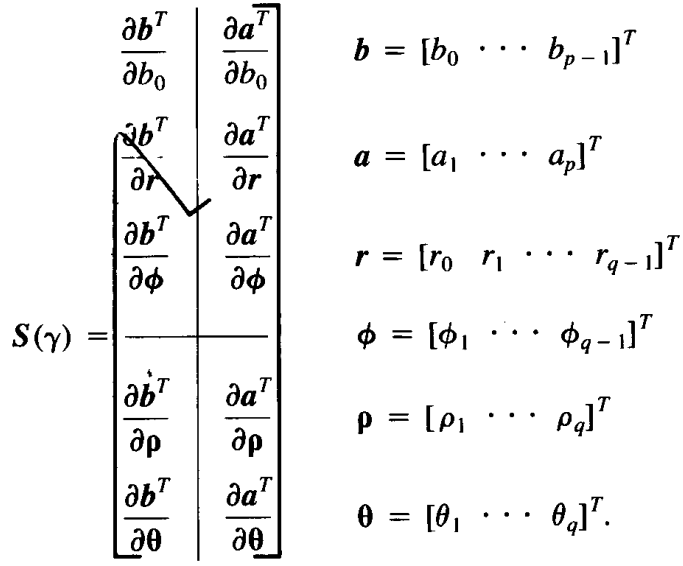


The transformation $\theta=g^{-1}(\gamma)$ implies that the northeast and southeast partitions of $\boldsymbol{S}(\gamma)$ are zero. Expressions for the terms in the southeast partition of $S(\gamma)$ have been derived in the section on the pole-residue characterization and can be calculated using (4.15) and (4.16). The terms in the northwest block are obtained from (4.31) using techniques similar to those already illustrated and they are stated here without derivation. We have defined $m$ $=p / 2-1$ :

$-\frac{1}{2} \frac{\partial \boldsymbol{b}^{T}}{\partial \boldsymbol{r}} \boldsymbol{B}^{-1}=$
$\cdot\left[\begin{array}{ccccc}0 & 1 / 2 & r_{0} / 2 & \cdots & r_{0}^{p-2} / 2 \\ 0 & \cos \phi_{1} & r_{1} \cos 2 \phi_{1} & \cdots & r_{1}^{p-2} \cos \left((p-1) \phi_{1}\right) \\ \vdots & \vdots & \vdots & & \vdots \\ 0 & \cos \phi_{m} & r_{m} \cos 2 \phi_{m} & \cdots & r_{m}^{p-2} \cos \left((p-1) \phi_{m}\right)\end{array}\right]$

$$
\frac{1}{2} \frac{\partial b^{T}}{\partial \phi} B^{-1}=
$$$$
\cdot\left[\begin{array}{ccccc}
0 & r_{1} \sin \phi_{1} & r_{1}^{2} \sin 2 \phi_{1} & \cdots & r_{1}^{p-1} \\
\vdots & \vdots & \vdots & \sin \left((p-1) \phi_{m}\right) \\
0 & r_{m} \sin \phi_{m} & r_{m}^{2} \sin 2 \phi_{m} \cdots r_{m}^{p-1} \sin \left((p-1) \phi_{m}\right)
\end{array}\right]
$$

sity estimators typically operate on a snapshot $y$ of an infinite time series $\left\{y_{t}\right\}_{0}^{\infty}$ which has infinite energy. The CR bounds for the stochastic model have been computed in [19] and [20] and these results differ from those we derive for the deterministic model of Fig. 3.

The map from the ARMA parameters to the energy spectrum is not, in general, invertible. Consequently, we will compute sensitivity matrices according to (2.1) and not from (2.3) which was utilized in the previous two sections.

The energy spectrum

$$
S\left(e^{j \theta} ; \boldsymbol{\theta}\right)=\left.S(z ; \theta)\right|_{z=e^{j \theta}}=\left.\frac{B(z)}{A(z)} \frac{B\left(z^{-1}\right)}{A\left(z^{-1}\right)}\right|_{z=e^{j \theta}}
$$

is a function of the frequency variable $\theta$ and, consequently, so is the $(2 p \times 1)$ sensitivity matrix $\boldsymbol{T}(\boldsymbol{\theta})$. Thus we must compute a new $\boldsymbol{T}(\boldsymbol{\theta})$ for every value of the energy spectrum we wish to bound.

The sensitivity matrix $\boldsymbol{T}(\boldsymbol{\theta})$, evaluated at frequency $\theta$, is

$$
\boldsymbol{T}(\boldsymbol{\theta})=\left[\frac{\partial S(z ; \boldsymbol{\theta})}{\partial b_{1}} \cdots \frac{\partial S(z ; \boldsymbol{\theta})}{\partial b_{p-1}} \frac{\partial S(z ; \boldsymbol{\theta})}{\partial a_{1}} \cdots \frac{\partial S(z ; \boldsymbol{\theta})}{\partial a_{p}}\right]_{z=e^{j \boldsymbol{\theta}}}^{T}
$$

$$
\boldsymbol{B}=\left[\begin{array}{cccc}
b_{0} & b_{1} & \cdots & b_{p-1} \\
& b_{0} & \cdots & b_{p-2} \\
& & & \vdots \\
0 & & & b_{0}
\end{array}\right] .
$$

\section{Sensitivity Matrix for the Energy Spectrum}

In the preceding sections we have derived sensitivity matrices for the parameters $\gamma$ which characterize the modal signal $\boldsymbol{x}$. The purpose of this section is to compute the sensitivity matrix that allows the CR bound to be computed for unbiased estimators of the energy spectrum. The term energy spectral density, rather than power spectral density, is used because the data available to the estimator are assumed to consist of a deterministic modal signal $\boldsymbol{x}$ and an additive Gaussian noise signal $\boldsymbol{n}$ as shown in Fig. 3. Since we have modeled $\left\{x_{t}\right\}$ as the impulse response of a stable ARMA system $B(z) / A(z)$, the modal signal $\left\{x_{t}\right\}_{0}^{\infty}$ has finite energy. In contrast, power spectral den-
This matrix has components which are invariant to time reversal, as shown below:

$$
\begin{aligned}
\frac{\partial S(z ; \boldsymbol{\theta})}{\partial b_{i}} & =\frac{1}{A(z) A\left(z^{-1}\right)}\left[z^{-i} B\left(z^{-1}\right)+z^{i} B(z)\right] \\
& =\frac{\partial S\left(z^{-1} ; \boldsymbol{\theta}\right)}{\partial b_{i}} \\
\frac{\partial S(z ; \boldsymbol{\theta})}{\partial a_{i}} & =\frac{-B(z) B\left(z^{-1}\right)}{A^{2}(z) A^{2}\left(z^{-1}\right)}\left[z^{-i} A\left(z^{-1}\right)+z^{i} A(z)\right] \\
& =\frac{\partial S\left(z^{-1} ; \boldsymbol{\theta}\right)}{\partial a_{i}} .
\end{aligned}
$$

This result has also been used in [20] to compute asympthe stochastic ARMA time series. We can simplify the form of the sensitivity matrix by letting it be partly composed of two Jury matrices (see (3.22)). The Jury matrix $M_{P}$ for a polynomial $P(z)=\sum_{i=0}^{n-1} p_{i} z^{-i}$ can be defined totic CR bounds for estimators of the power spectrum for 
implicitly by

$$
\begin{aligned}
M_{P}\left[\Psi_{n}\left(z^{-1}\right)+\Psi_{n}(z)\right] & =P\left(z^{-1}\right) \psi_{n}\left(z^{-1}\right)+P(z) \Psi_{n}(z) \\
& =M_{P} v_{n}(z)
\end{aligned}
$$

where $\psi_{n}(z)=\left[\begin{array}{llll}1 & z & \cdots & z^{n-1}\end{array}\right]^{T}$. Equations (4.40) through (4.42) indicate that we can write the sensitivity matrix as

$$
\boldsymbol{T}=\left[\begin{array}{l}
\left(\left|H\left(e^{j \theta}\right)\right|^{2}\right) \boldsymbol{M}_{B} \boldsymbol{v}_{p}\left(e^{j \theta}\right) \\
\left(-\left|K\left(e^{j \theta}\right)\right|^{2}\right) \boldsymbol{W M}_{A} \boldsymbol{v}_{p+1}\left(e^{j \theta}\right)
\end{array}\right]
$$

where $W=\left[\begin{array}{ll}0 & I\end{array}\right]$ and the transfer functions $H(z)=$ $1 / A(z)$ and $K(z)=-B(z) / A^{2}(z)$ correspond to those in (3.15).

We now have a means of determining $T(\theta)$ at any frequency $\theta$, and the CR bound for any unbiased estimator $\hat{S}\left(e^{j \theta}\right)$ is

$$
E\left\{\left(\hat{S}\left(e^{j \theta}\right)-S\left(e^{j \theta} ; \boldsymbol{\theta}\right)\right)^{2}\right\} \geq \boldsymbol{T}^{T}(\boldsymbol{\theta}) \boldsymbol{J}^{-1}(\boldsymbol{b}, \boldsymbol{a}) \boldsymbol{T}(\boldsymbol{\theta}) .
$$

By sweeping $\theta$ over the interval $[0, \pi],(4.44)$ can be used to generate CR bounds for the entire energy spectrum. We can also investigate the effects of simultaneously estimating two or more values of the spectrum by making the following modifications. Let $S=\left[S\left(e^{j \theta_{1}}\right) S\left(e^{j \theta_{2}}\right) \cdots\right.$ $\left.S\left(e^{j \theta_{m}}\right)\right]^{T}$ be a vector of the spectrum values at $m$ frequencies of interest. The error covariance matrix for this vector has the CR bound

$$
E\left\{(\hat{\boldsymbol{S}}-\boldsymbol{S})(\hat{\boldsymbol{S}}-\boldsymbol{S})^{T}\right\} \geq \boldsymbol{T}^{T} \boldsymbol{J}^{-1}(\boldsymbol{b}, \boldsymbol{a}) \boldsymbol{T}
$$

where the $(2 p \times m)$ sensitivity matrix $\boldsymbol{T}$ is composed of the $(2 p \times 1)$ sensitivity matrices $T_{i}$ for each frequency $\theta_{i}$ :

$$
\boldsymbol{T}^{T}=\left[\begin{array}{c}
\boldsymbol{T}_{1}^{T} \\
\boldsymbol{T}_{2}^{T} \\
\vdots \\
\boldsymbol{T}_{m}^{T}
\end{array}\right]
$$

The sensitivity matrix characterized in equation (4.43) together with the Fisher matrices $J(b, a)$ derived in (3.8) and (3.16) complete our theoretical treatment of CR bounds on unbiased estimators of the energy spectrum $S\left(e^{j \theta} ; \boldsymbol{\theta}\right)$.

\section{Numerical Results}

Cramer-Rao bounds afford an effective method for evaluating estimator performance. In the context of modal analysis, they also provide a way to quantitatively describe which properties of the modal signal have the greatest (or least) effect on the ability to estimate the mode parameters. Concentration ellipses, generated from the Fisher information matrix, can provide additional insight into the interactions among estimation errors. In the remainder of this section we will use these tools on a few illustrative examples to investigate some of the properties of modal parameter estimation.

\section{A. Single Mode}

The first modal signal to be analyzed consists of a single damped mode with arbitrary amplitude and phase. The time domain description of the modal signal

$$
x_{t}=A \rho^{t} \cos (\theta t+\phi)
$$

is characterized by the pole-residue parameters $A$ (amplitude), $\phi$ (phase), $\rho$ (damping coefficient), and $\theta$ (frequency). The sensitivity matrix for the pole-residue parameterization is derived by equating, in the transform domain, the ARMA description and pole-residue description for $X(z)=B(z) / A(z)$ :

$$
\begin{aligned}
\frac{B(z)}{A(z)} & =\frac{b_{0}+b_{0} z^{-1}}{1+a_{1} z^{-1}+a_{2} z^{-2}} \\
& =\frac{(A / 2) e^{j \phi}}{1-\rho e^{j \theta} z^{-1}}+\frac{(A / 2) e^{-j \phi}}{1-\rho e^{-j \theta} z^{-1}} \\
& =\frac{A \cos (\phi)-A \rho \cos (\theta-\phi) z^{-1}}{1-2 \rho \cos (\theta) z^{-1}+\rho^{2} z^{-2}} .
\end{aligned}
$$

All the results for this example are generated for the asymptotic case, using the Fisher matrix of (3.16) and the sensitivity matrix of Section IV-A, and are presented in the form of the concentration ellipses in Figs. 4 and 5 and the CR bound plot in Fig. 6. The concentration ellipses were generated assuming that the noise variance $\sigma^{2}$ was equal to 1 . Increasing $\sigma^{2}$ will increase the volume of the concentration ellipses, but the relative shapes and interactions of the ellipses will remain constant.

The effects of varying the amplitude parameter $A$ can be observed in Figs. 4(a) and 5(a). Note that in all cases the volume of the concentration ellipses is reduced as the amplitude parameter is increased. As stated in Section II, the concentration ellipse for the estimator error covariance matrix $C$ encloses (is lower bounded by) the concentration ellipses derived from the Fisher information matrix. Therefore we can state that increasing the mode amplitude $A$ reduces, except for the error $e(A)$, the bounds on the error variances for the mode parameters. Figs. 4(a) and 5(a) exhibit a phenomenon we have designated "pinning." Pinning occurs when the ellipses intersect at common points along a zero axis. When a set of ellipses are pinned on the horizontal axis, then the parameter being varied has no effect on the estimator error for the parameter corresponding to the pinned axis if all other parameters are known. For example, in Fig. 4(a) the ellipses are pinned on the $e(A)$ axis. This implies that, if the parameters $\phi, \rho$, and $\theta$ are known, then the estimator error $e(A)$ is not influenced by the amplitude parameter $A$. (Of course, the fractional error $e(A) / A$ decreases with increasing $A$, and this is what counts!) It is not hard to show that the bound on the error variance for the amplitude parameter $A$ is independent of the value of $A$, meaning that 


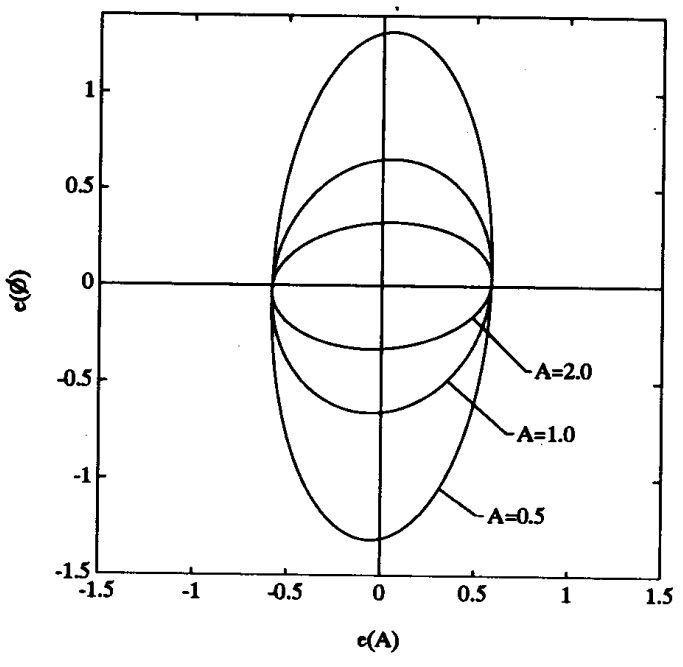

(a)

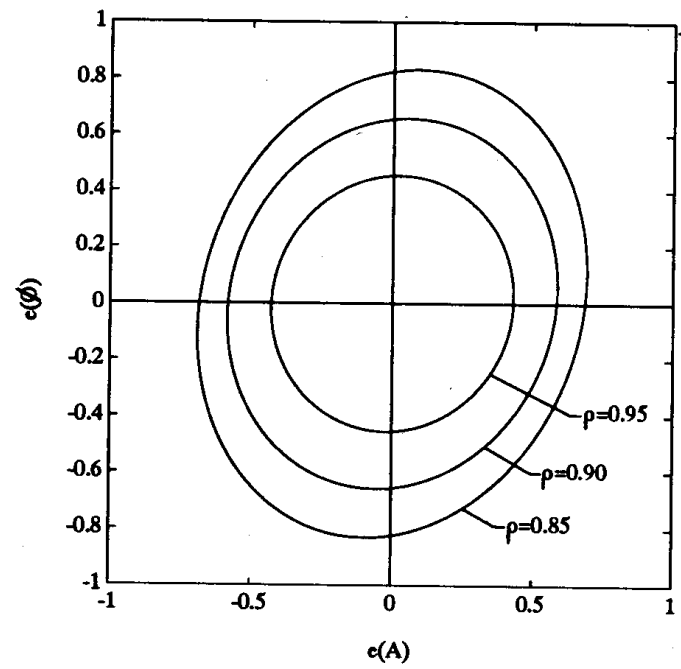

(c)

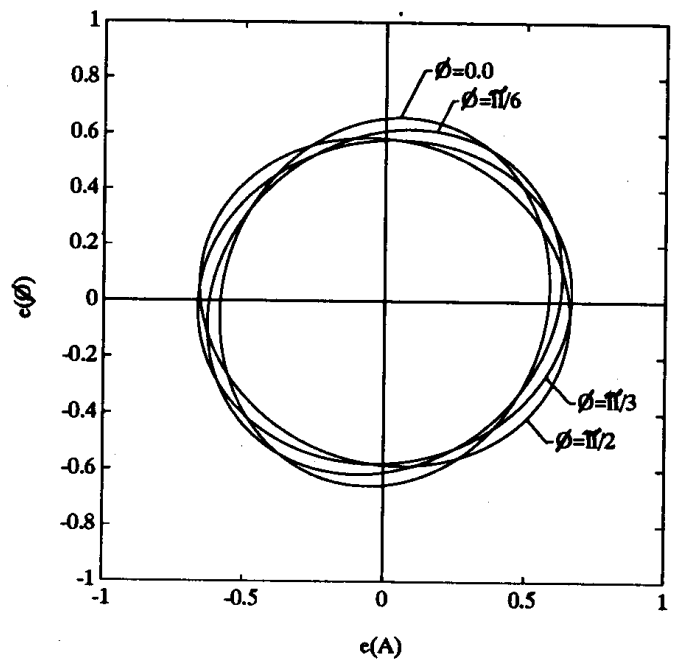

(b)

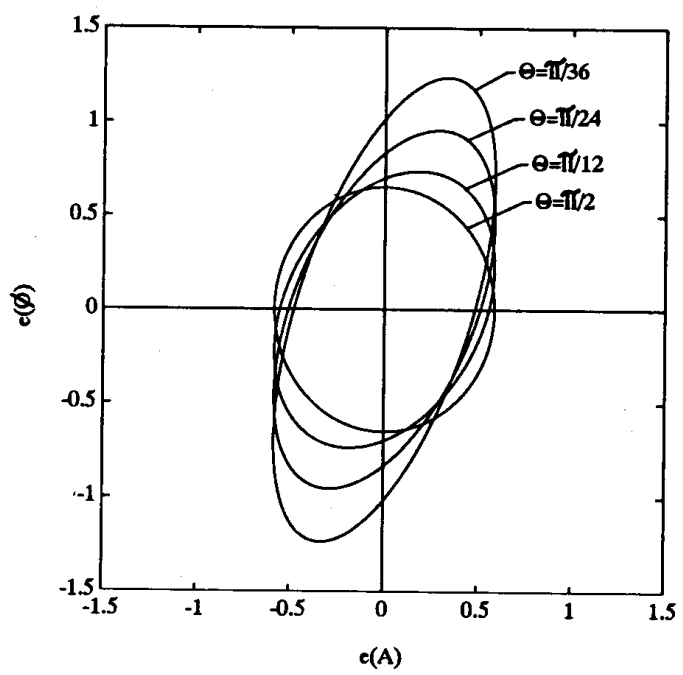

(d)

Fig. 4. Concentration ellipses for $(A, \phi)$. (a) $A=$ var, $\phi=0.0, \rho=0.9, \theta=\pi / 4.0$. (b) $A=1.0, \phi=$ var, $\rho=0.9, \theta=$ $\pi / 4.0$. (c) $A=1.0, \phi=0.0, \rho=\operatorname{var}, \theta=\pi / 4.0$. (d) $A=1.0, \phi=0.0, \rho=0.9, \theta=$ var

the variance of $e(A) / A$ decreases in proportion to $1 / A^{2}$. The variance bounds on the remaining parameters $\phi, \rho$, and $\theta$ reduce in proportion to $1 / A^{2}$ as well. The effects of varying the phase parameter $\phi$ can be observed in Figs. 4(b) and 5(b). These ellipses illustrate that the phase offset has little influence on the ability to estimate mode parameters for the single mode case. The pinning phenomenon is not observed in Fig. 4(b).

The effects of varying the damping coefficient $\rho$ can be seen in Figs. 4(c) and 5(c). Without exception, as $\rho \rightarrow 1$ the CR bounds on the modal parameters decrease significantly as evidenced in the magnitude of the ellipse reductions. The explanation for this property is that, for larger $\rho$, the energy in the modal signal $\boldsymbol{x}$ increases, and more samples are obtained before $x_{t}$ is damped to a value low in comparison with the noise. Since the ellipses for larger $\rho$ are entirely contained within the ellipses for smaller $\rho$, we can state that $J_{\rho_{1}}^{-1}<J_{\rho 2}^{-1}$ if $\rho_{1}>\rho_{2}$.

The effects of varying the frequency $\theta$ of a mode can be observed in Figs. 4(d) and 5(d). These figures indicate that the value of $\theta$ has the most significant effect on the phase error $e(\phi)$. The phase estimation error is minimized in all cases as $\theta \rightarrow \pi / 2$. The estimation errors for the remaining parameters are not significantly influenced by $\theta$ until $\theta \rightarrow 0$ or $\theta \rightarrow \pi$. Fig. 6 illustrates in a different manner the influence of $\theta$ on the CR bounds for the modal parameters. The bounds were calculated with $A=1, \rho=$ $0.9, \phi=0$, and variable $\theta$. The noise variance $\sigma^{2}$ was normalized to 1 . The bounds can be found for any other noise variance by adding $10 \log _{10}\left(\sigma^{2}\right)$ to these curves. 


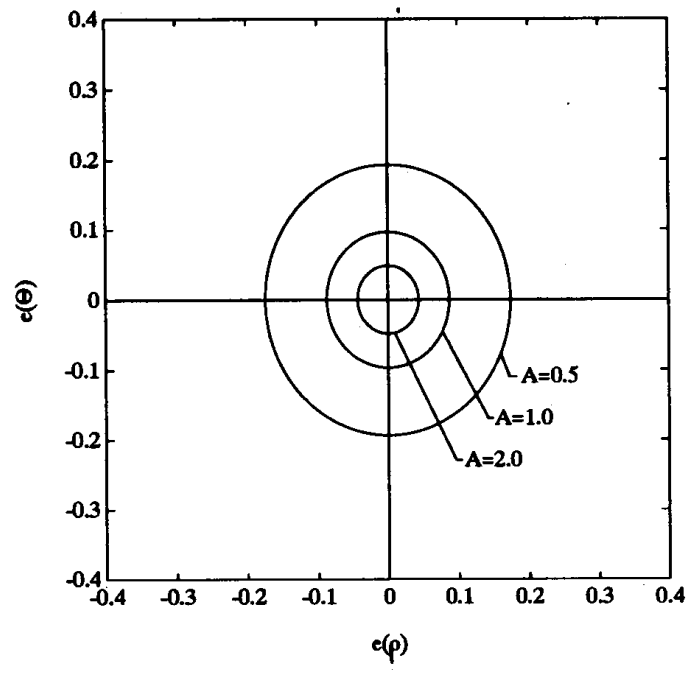

(a)

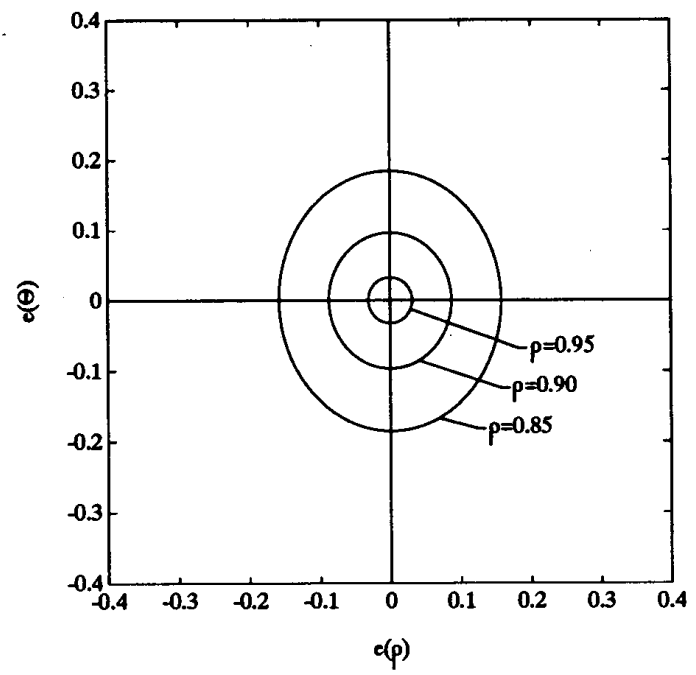

(c)

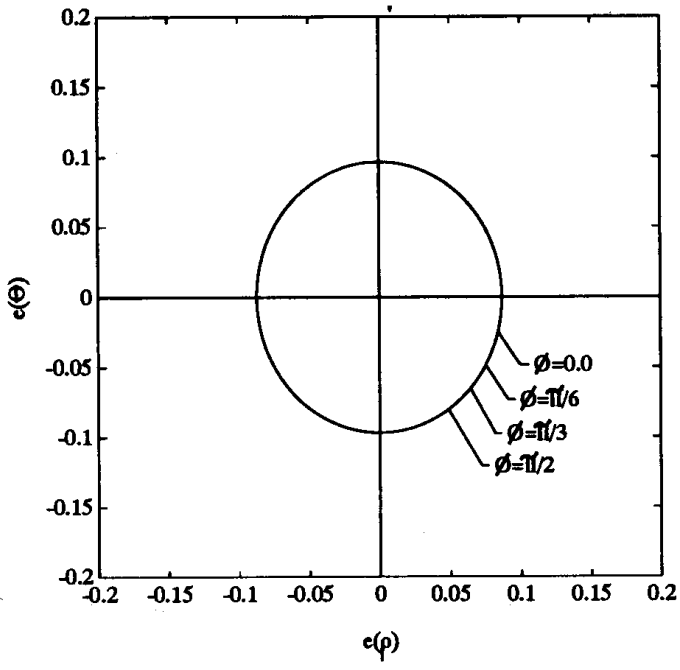

(b)

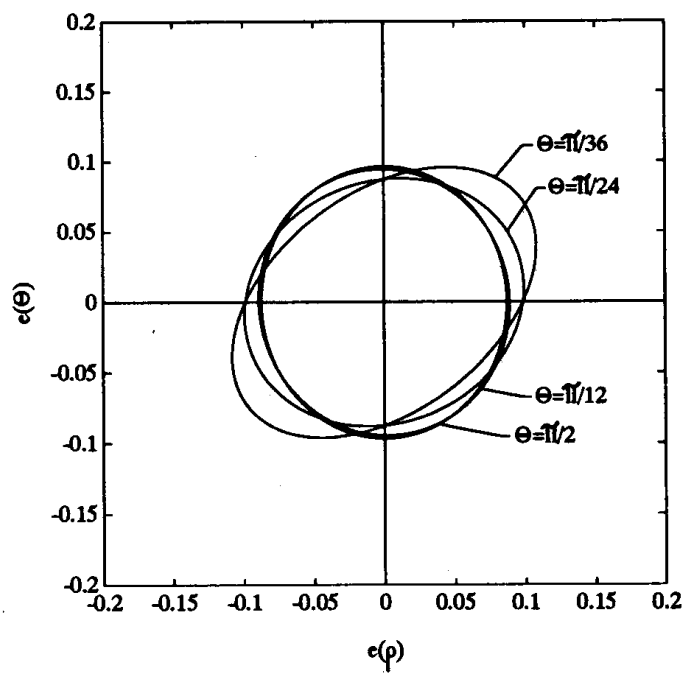

(d)

Fig. 5. Concentration ellipses for $(\rho, \theta)$. (a) $A=\operatorname{var}, \phi=0.0, \rho=0.9, \theta=\pi / 4.0$. (b) $A=1.0, \bar{\phi}=$ var, $\rho=0.9, \theta=$ $\pi / 4.0$. (c) $A=1.0, \phi=0.0, \rho=\operatorname{var}, \theta=\pi / 4.0$, (d) $A=1.0, \phi=0.0, \rho=0.9, \theta=$ var.

This figure indicates that as $\theta \rightarrow 0$ or $\theta \rightarrow \pi$ the CR bounds for the modal parameters show dramatic increases. We attribute this phenomenon to the close proximity of the conjugate poles $z=\rho e^{j \theta}$ and $z=\rho e^{-j \theta}$ as $\theta \rightarrow 0$ or $\theta \rightarrow$ $\pi$. The pole proximity effects will be discussed in the following section.

\section{B. Dual Modes}

In this section a modal signal consisting of two independent modes is analyzed. Since the effects of mode amplitude are easily determined, the modes in this example have unit amplitude. The phase offset for each mode is set to zero for convenience. With these constraints in force, the modal signal

$$
x_{t}=\rho_{1}^{t} \cos \left(\theta_{1} t\right)+\rho_{2}^{t} \cos \left(\theta_{2} t\right)
$$

can be characterized by the frequencies $\theta_{1}$ and $\theta_{2}$ and the damping coefficients $\rho_{1}$ and $\rho_{2}$ of the two modes. It will be convenient for subsequent discussions to also characterize the modal signal in terms of the mean frequency $\theta_{m}$ $=\left(\theta_{1}+\theta_{2}\right) / 2$ and the difference or beat frequency $\theta_{d}=$ $\left|\theta_{2}-\theta_{1}\right| / 2$ :

$$
x_{t}=\rho_{1}^{t} \cos \left(\left(\theta_{m}-\theta_{d}\right) t\right)+\rho_{2}^{t} \cos \left(\left(\theta_{m}+\theta_{d}\right) t\right) .
$$

The sensitivity matrix $S(\gamma)$ for the modal parameters $\gamma^{T}$ $=\left[\begin{array}{llll}\rho_{1} & \rho_{2} & \theta_{m} & \theta_{d}\end{array}\right]$ is derived by equating, in the frequency domain, the ARMA characterization and pole-res- 


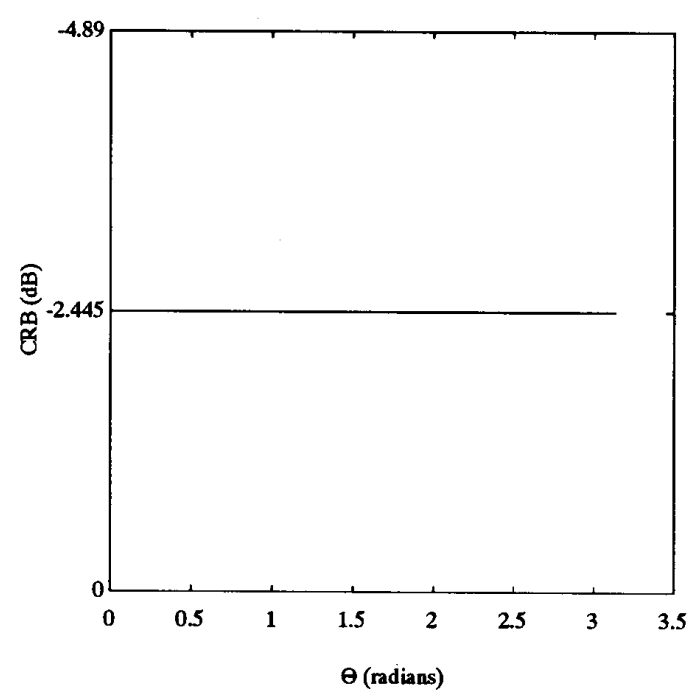

(a)

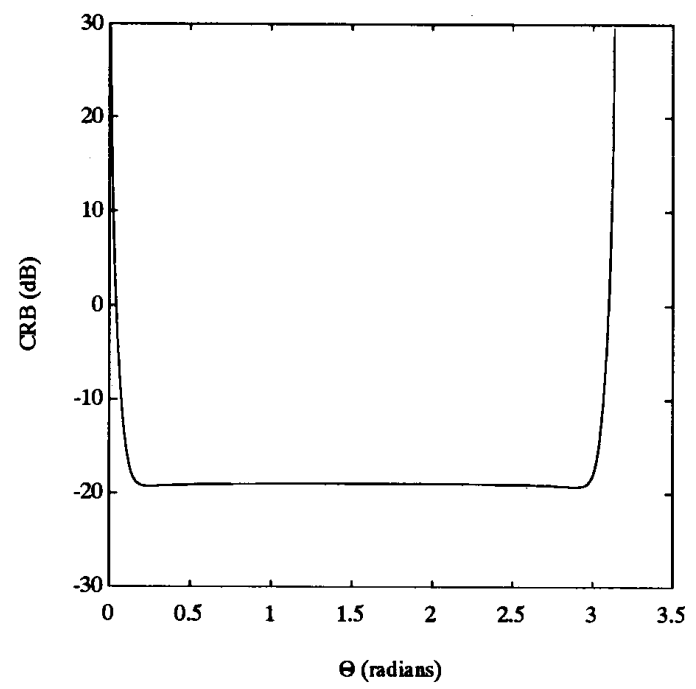

(c)

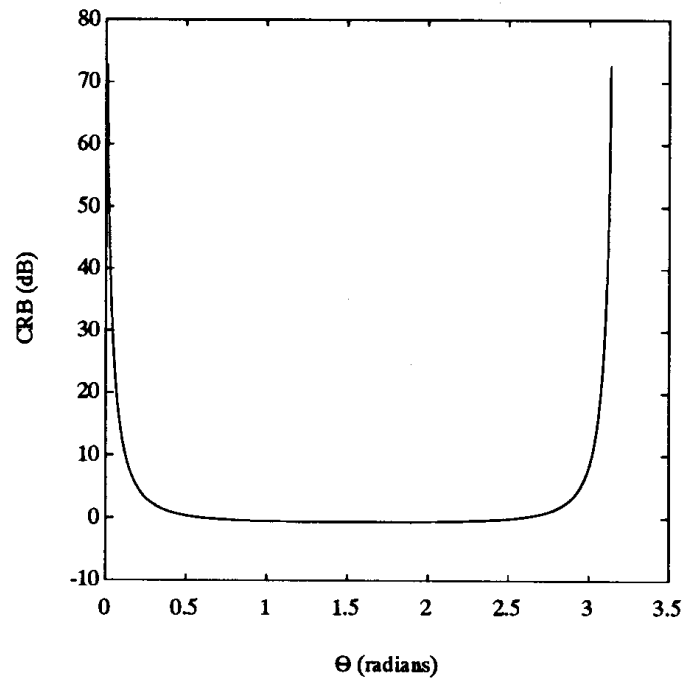

(b)

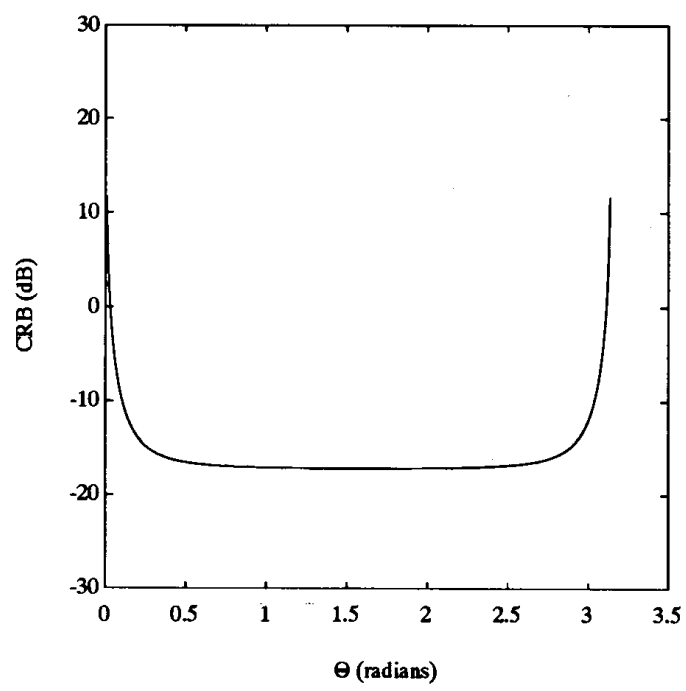

(d)

Fig. 6. CR bounds versus frequency $\theta$. (a) Normalized CRB for $A$ versus $\theta$. (b) Normalized CRB for $\phi$ versus $\theta$. (c) Normalized CRB for $\rho$ versus $\theta$. (d) Normalized CRB for $\theta$ versus $\theta$.

idue parameterization of the modal signal $x_{1}$ :

$$
\begin{aligned}
\frac{B(z)}{A(z)}= & \frac{b_{0}+b_{1} z^{-1}+b_{2} z^{-2}+b_{3} z^{-3}}{1+a_{1} z^{-1}+a_{2} z^{-2}+a_{3} z^{-3}+a_{4} z^{-4}} \\
= & \frac{1-\rho_{1} \cos \left(\theta_{m}-\theta_{d}\right) z^{-1}}{1-2 \rho_{1} \cos \left(\theta_{m}-\theta_{d}\right) z^{-1}+\rho_{1}^{2} z^{-2}} \\
& +\frac{1-\rho_{2} \cos \left(\theta_{m}+\theta_{d}\right) z^{-1}}{1-2 \rho_{2} \cos \left(\theta_{m}+\theta_{d}\right) z^{-1}+\rho_{2}^{2} z^{-2}} .
\end{aligned}
$$

The locations of the poles of the modal signal are illustrated in Fig. 7 in terms of the mode parameters $\gamma^{T}=$ $\left[\begin{array}{llll}\rho_{1} & \rho_{2} & \theta_{m} & \theta_{d}\end{array}\right]$.
In this section, we will use this model to investigate the effects of mode spacing on CR bounds for modal parameters. Fig. 8 is generated for infinite data with the mode parameters $\rho_{1}=\rho_{2}=0.9, \theta_{m}=\pi / 2$, and $\theta_{d}$ variable. As in Fig. 6, the noise variance was normalized to one and CR bounds for different $\sigma^{2}$ can be found as described in the latter part of Section V-A. It is evident from Fig. 8 that, as $\theta_{d} \rightarrow 0$ (i.e., the mode frequencies $\theta_{1}$ and $\theta_{2}$ approach each other), the mode parameters become harder to identify. Note that this pole proximity effect is stronger for the beat frequency $\theta_{d}$ than for the mean frequency $\theta_{m}$. As $\theta_{d} \rightarrow \pi / 2$, the conjugate poles for mode $1, z_{1}=$ $\rho_{1} e^{j \theta_{1}}$ and $z_{1}^{*}=\rho_{1} e^{-j \theta_{1}}$, approach each other on the real axis at $\rho_{1}$. The conjugate poles for mode 2 approach each 


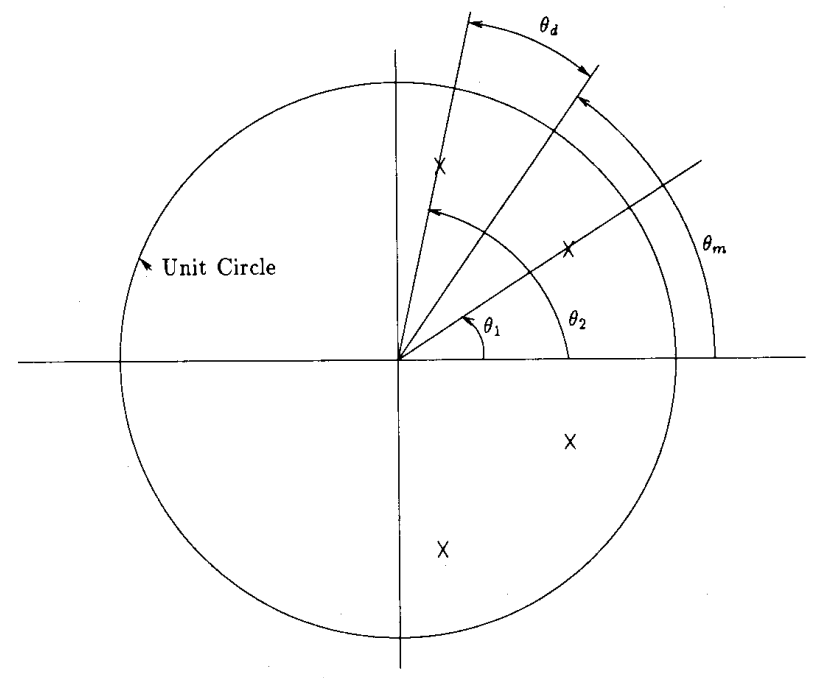

Fig. 7. Geometry of pole locations.
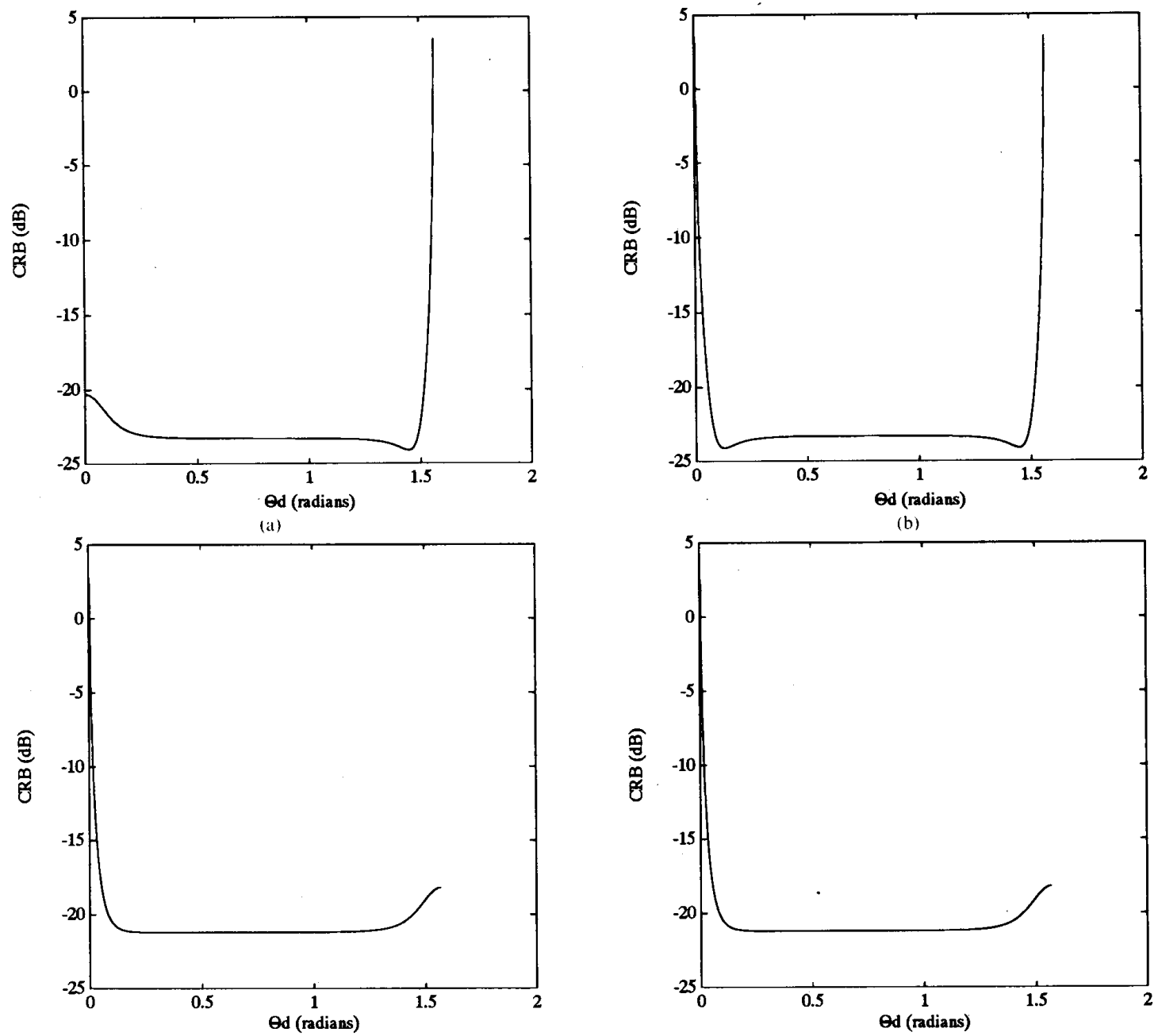

(d)

Fig. 8. CR bounds versus difference frequency. (a) Normalized CRB for $\rho_{1}$ versus $\theta_{\iota}: \theta_{t \prime}=\pi / 2$. (b) Normalized CRB for $\rho_{2}$ versus $\theta_{d l}: \theta_{m}=\pi / 2$. (c) Normalized CRB for $\theta_{m}$ versus $\theta_{d l}: \theta_{m}=\pi / 2$. (d) Normalized CRB for $\theta_{d}$ versus $\theta_{d}: \theta_{m}=\pi / 2$. 


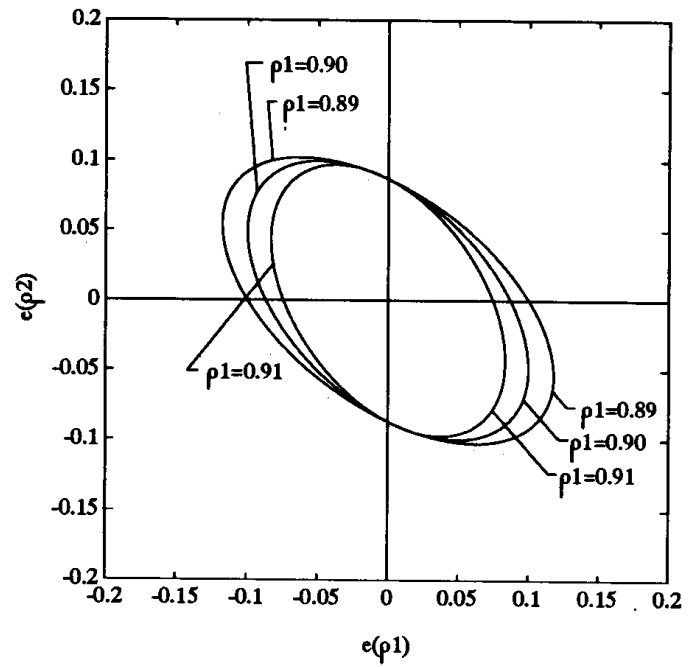

(a)

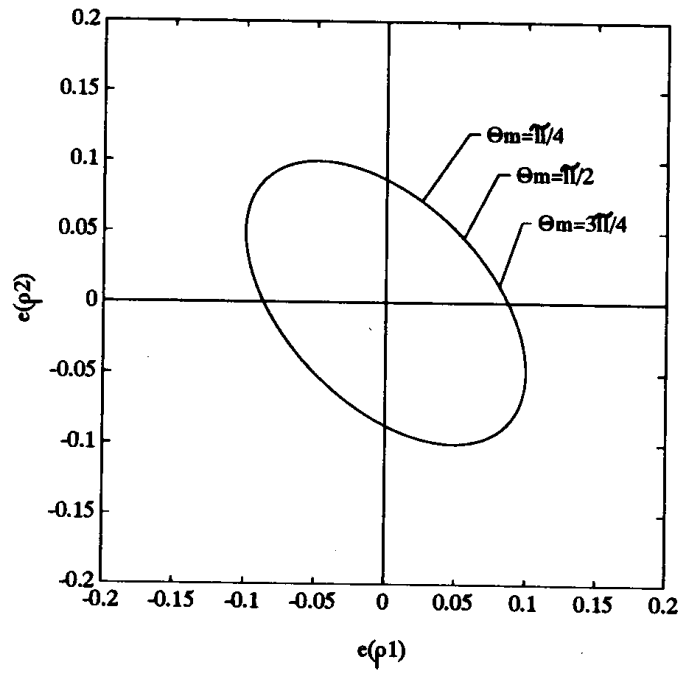

(c)

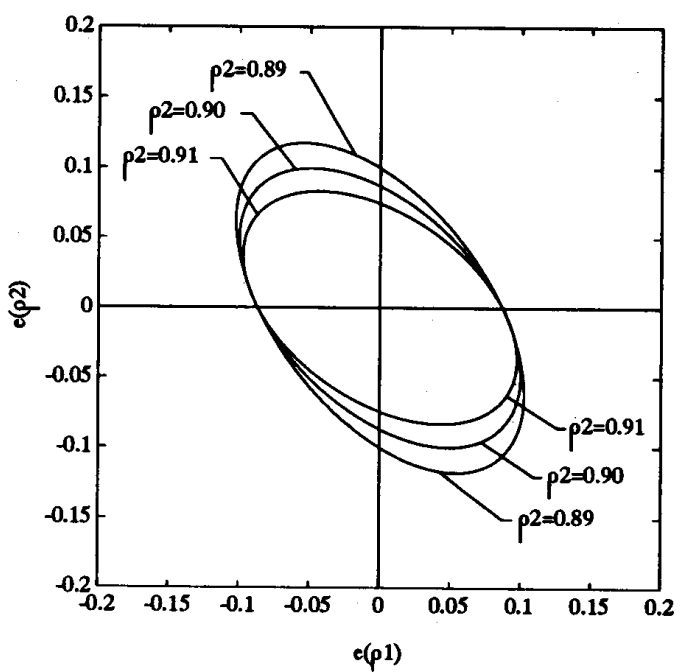

(b)

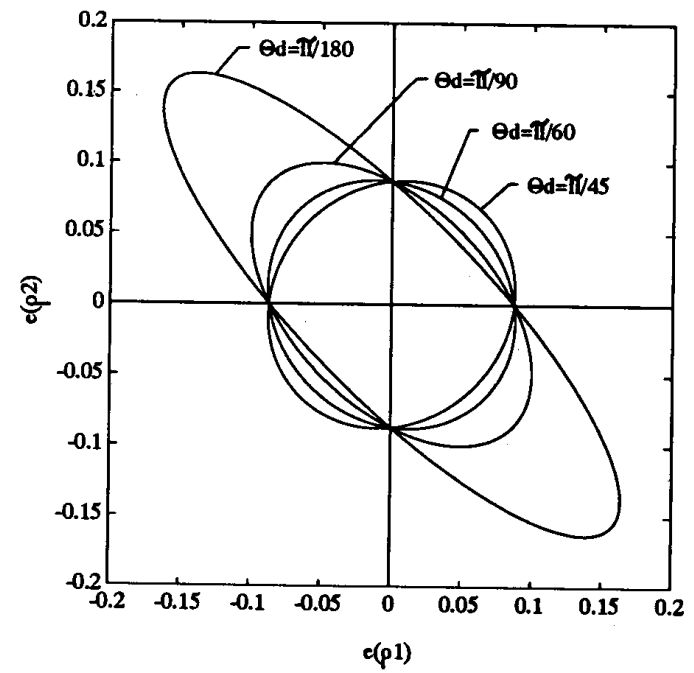

(d)

Fig. 9. Concentration ellipses for $\left(\rho_{1}, \rho_{2}\right)$. (a) $\rho_{1}=$ var, $\rho_{2}=0.90, \theta_{m}=\pi / 2, \theta_{d}=\pi / 90$. (b) $\rho_{1}=0.90, \rho_{2} \pm$ var, $\theta_{m}=$ $\pi / 2, \theta_{d}=\pi / 90$. (c) $\rho_{1}=0.90, \rho_{2}=0.90, \theta_{m}=\operatorname{var}, \theta_{d}=\pi / 90$. (d) $\rho_{1}=0.90, \rho_{2}=0.90, \theta_{m}=\pi / 2, \theta_{d}=$ var.

other on the real axis at $-\rho_{2}$. Again we observe the conjugate pole proximity effects described in Section V-A. The concentration ellipses in Figs. 9 and 10 can be used to examine these proximity effects and also the interaction of estimation errors. These ellipses, like those in Figs. 4 and 5 , were generated with $\sigma^{2}$ normalized to one.

The consequences of varying the mode parameters $\rho_{1}$ and $\rho_{2}$ can be observed in Figs. 9 and 10, subplots (a) and (b), respectively. These results correspond closely to those obtained for the single mode case. To reiterate, mode parameters are easier to identify for larger damping coefficients $\rho$. Fig. 9(a) can be used to determine, for this mode spacing, that the size of the damping coefficient has a greater effect on the concentration ellipse volume than does the proximity of the poles. With $\rho_{2}=0.9$, the con- centration ellipse for $\rho_{1}=0.89$ is larger than the concentration ellipse for $\rho_{1}=0.9$, even though the distance between the poles increases.

The concentration ellipses in Figs. 9(c) and 10(c) indicate that the mean frequency $\theta_{m}$ has no effect as long as $\theta_{1}$ and $\theta_{2}$ are sufficiently far away from 0 or $\pi$.

The effects of varying the beat frequency $\theta_{d}$ can be observed by examining the concentration ellipses of Figs. $9(\mathrm{~d})$ and $10(\mathrm{~d})$. This is equivalent to varying the distance between the modal frequencies. These ellipses confirm the observation made about Fig. 8: when two modes are closely spaced, it is easier to identify the mean frequency $\theta_{m}$ than the beat frequency $\theta_{d}$. Note that the ellipses of Fig. 10(d) have pinning points which lie on $\pm 45^{\circ}$ degree lines. These diagonal axes correspond to the axes for the 


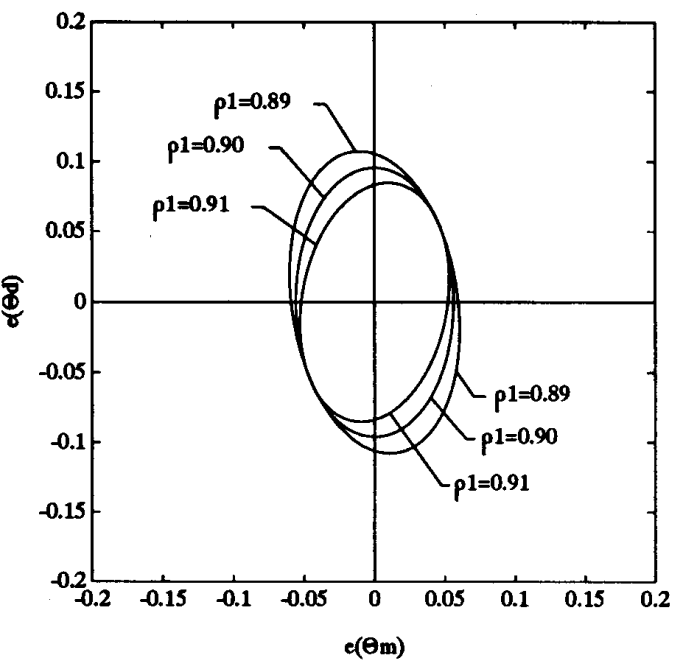

(a)

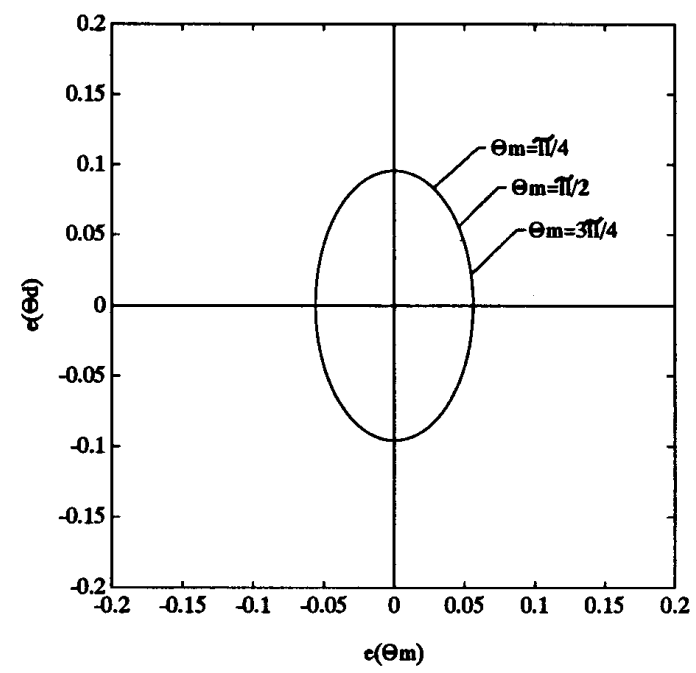

(c)

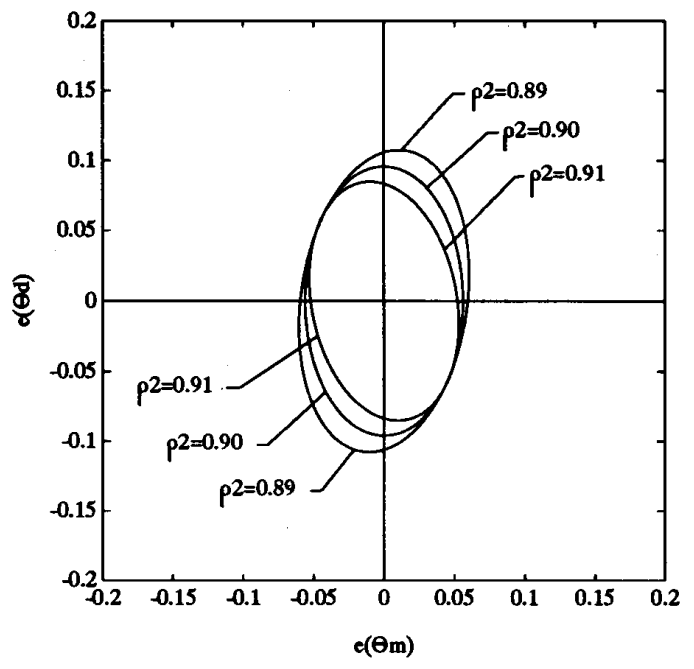

(b)

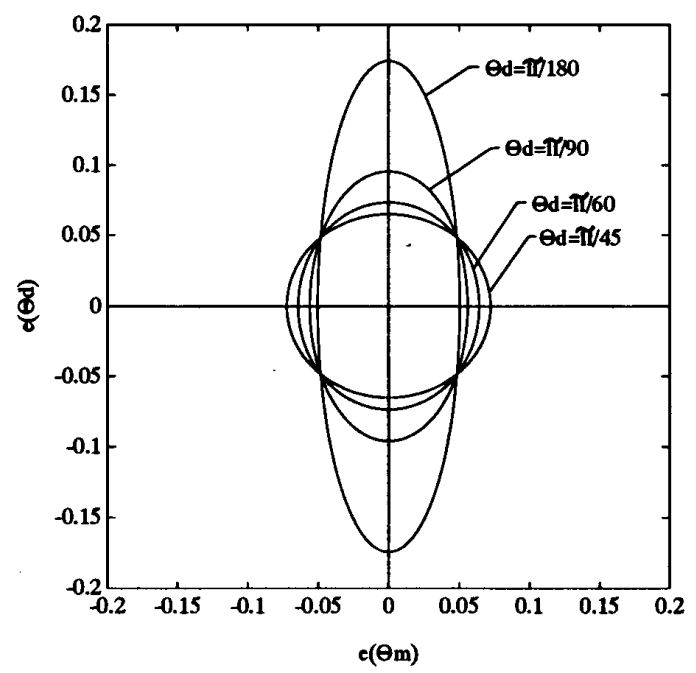

(d)

Fig. 10. Concentration ellipses for $\left(\theta_{m}, \theta_{d}\right)$. (a) $\rho_{1}=$ var, $\rho_{2}=0.90, \theta_{m}=\pi / 2, \theta_{d}=\pi / 90$. (b) $\rho_{1}=0.90, \rho_{2}=$ var, $\theta_{m}=$ $\pi / 2, \theta_{d}=\pi / 90$. (c) $\rho_{1}=0.90, \rho_{2}=0.90, \theta_{m}=\operatorname{var}, \theta_{d}=\pi / 90$. (d) $\rho_{1}=0.90, \rho_{2}=0.90, \theta_{m}=\pi / 2, \theta_{d}=$ var.

explicit mode frequencies $\theta_{1}$ and $\theta_{2}$. The pinning points indicate that, if the modal parameters $\theta_{1}, \rho_{1}$, and $\rho_{2}$ are known, then varying the distance $\theta_{d}$ between the frequencies has no effect on the error $e\left(\theta_{2}\right)$ in estimating $\theta_{2}$.

To explain the pole proximity effects for $\theta_{d} \rightarrow 0$, consider the case where $\rho_{1}=\rho_{2}=\rho$. The modal signal can now be expressed as

$$
x_{t}=2 \rho^{t} \cos \left(\theta_{d} t\right) \cos \left(\theta_{m} t\right) .
$$

When the beat frequency $\theta_{d}$ is small, the beat sinusoid $\cos$ $\left(\theta_{d} t\right)$ does not have a chance to significantly shape the modal signal $x_{t}$ before the damping term $\rho^{t}$ reduces the magnitude of $x_{t}$ to a value small in comparison to the noise variance. The explanation for the conjugate pole prox- imity effects is similar. Consider a single mode $x_{t}=\rho^{t}$ $\cos (\theta t)$ where $\theta$ is small (conjugate poles near real axis at $\theta$ ). Again, when $\theta$ is small, the sinusoid $\cos (\theta t)$ has little effect on the modal signal before the damping term has driven the magnitude of $x_{t}$ to an insignificant value compared to the noise variance. When $\theta \rightarrow \pi$ it is easy to show that $x_{t}=(-\rho)^{t} \cos \left(\theta_{s} t\right)$ where $\theta_{s}=\pi-\theta$ is small, and the same explanation applies.

\section{Energy Spectra}

In this section we use the CR bounds for estimating the energy spectrum to study two types of problems: 1) estimation of the energy spectrum from infinite (but noisy) 


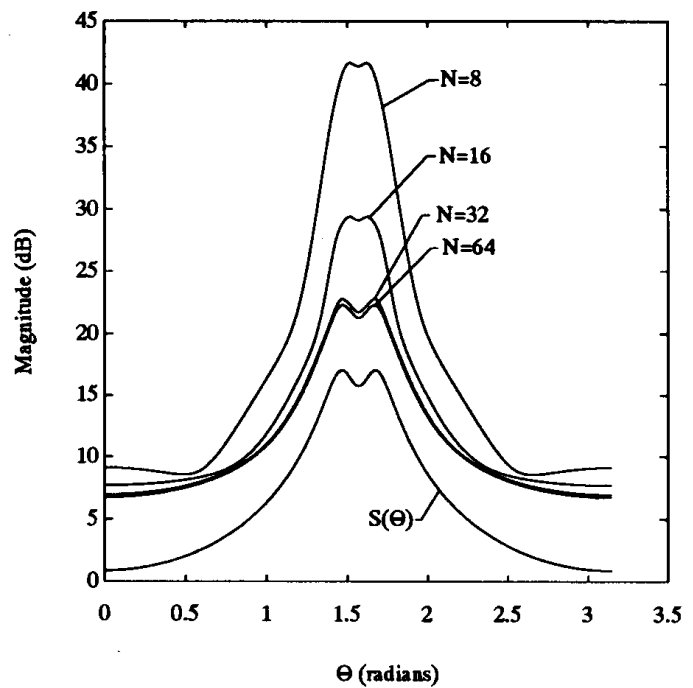

(a)

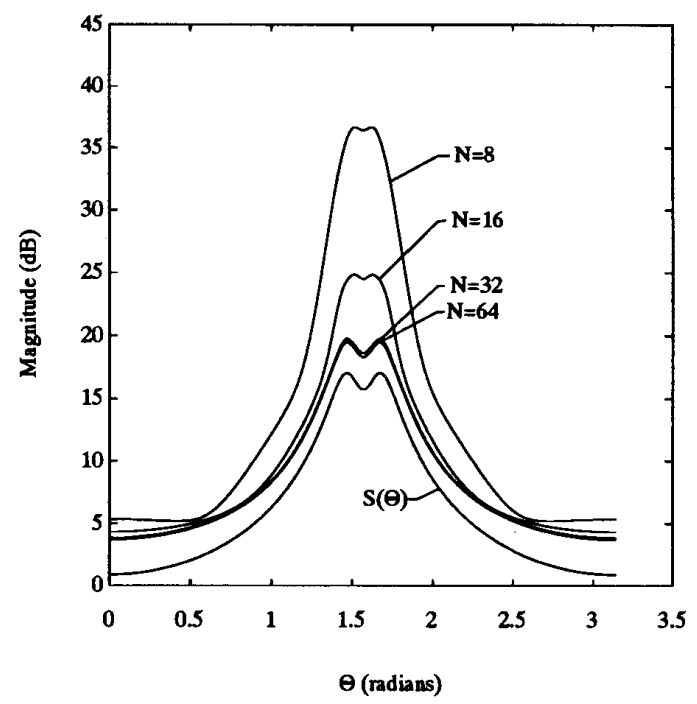

(c)

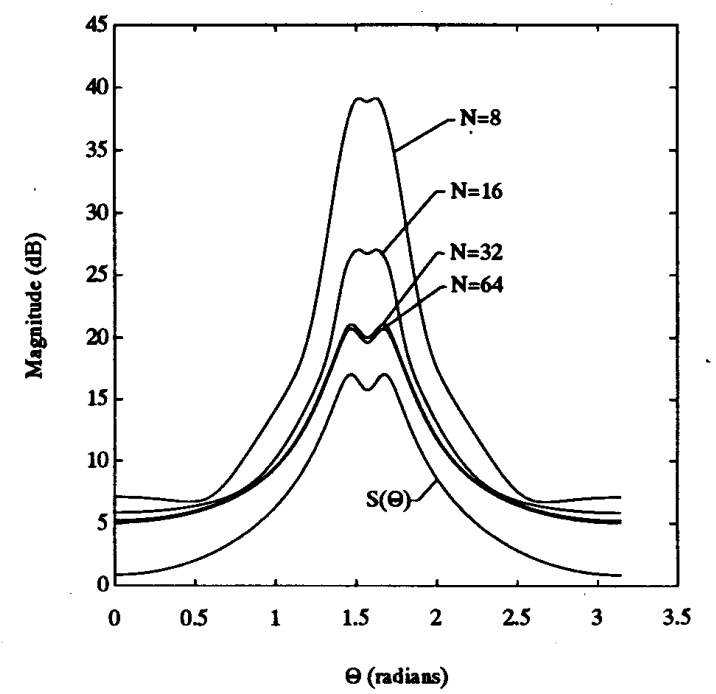

(b)

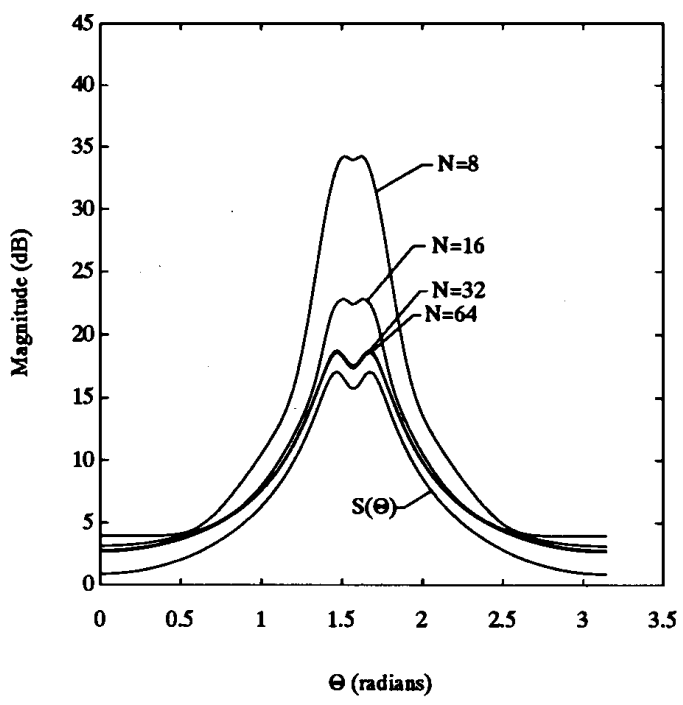

(d)

Fig. 11. CR bounds versus SNR for estimating a dual-mode energy spectrum with (a) $\mathrm{SNR}=5 \mathrm{~dB}$. (b) $\mathrm{SNR}=10 \mathrm{~dB}$, (c) SNR $=15 \mathrm{~dB}$, (d) SNR $=20 \mathrm{~dB}$.

data and (2) resolution of closely spaced spectral peaks from time-limited data. For both problems we choose the energy spectrum to be the spectrum for the dual mode signal studied in Section V-B.

The CR bounds of Fig. 11 show the dual mode spectrum $S\left(e^{j \theta}\right)$ for a fixed difference frequency of $2 \theta_{d}=$ $2 \pi / 32$. The CR bound is used to compute and plot $S\left(e^{j \theta}\right)$ $+\left[\operatorname{var}\left[S\left(e^{j \theta}\right)\right]\right]^{1 / 2}$ for various asymptotic SNR's and sample sizes $N$. These plots define a confidence interval about the actual spectrum $S\left(e^{j \theta}\right)$ if the lower bound of the confidence interval $S\left(e^{j \theta}\right)-\left[\operatorname{var}\left[S\left(e^{j \theta}\right)\right]\right]^{1 / 2}$ is included. We have omitted the lower bound to preserve clarity in the figure. Asymptotic signal to noise ratio is defined to be SNR $=\left(1 / \sigma^{2}\right) \Sigma_{0}^{\infty} x_{i}^{2}$. Fig. 11 indicates that even at
20-dB SNR and $N=64$ samples, any unbiased estimator cannot, on the average, resolve the spectral peaks present in this spectrum. The estimated spectrum will fall somewhere in the confidence band just discussed. This band is not small enough to ensure that the spectral peaks will be accounted for in the estimated spectrum. The Rayleigh limit to resolution for this mode spacing is $N=32$ samples. For sample sizes less than the Rayleigh limit, the size of the confidence interval shows a marked threshold effect. This threshold effect is perhaps better illustrated in Fig. 12 where the CR bounds are computed for the spectrum at one frequency corresponding to a spectral peak. The Rayleigh limit of $2 \pi / 32$ defines the sample size $N=$ 32 where the threshold effect is active. 


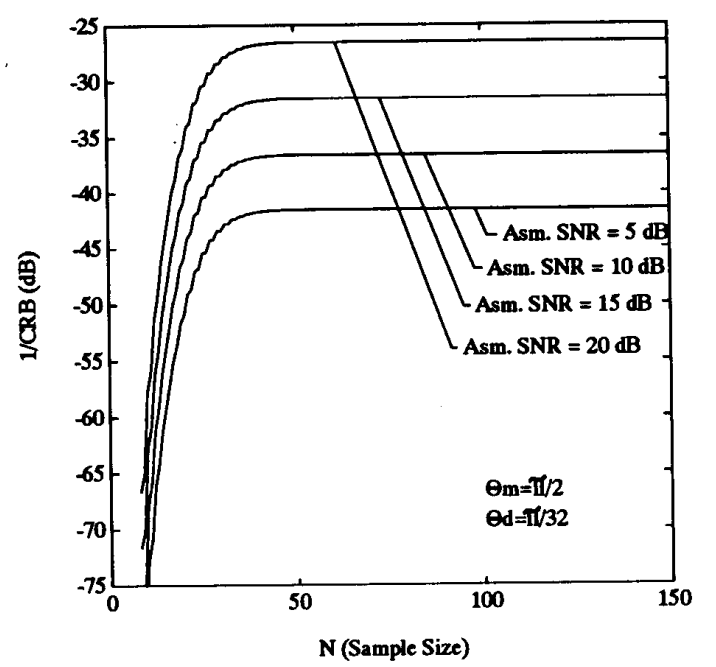

(a)

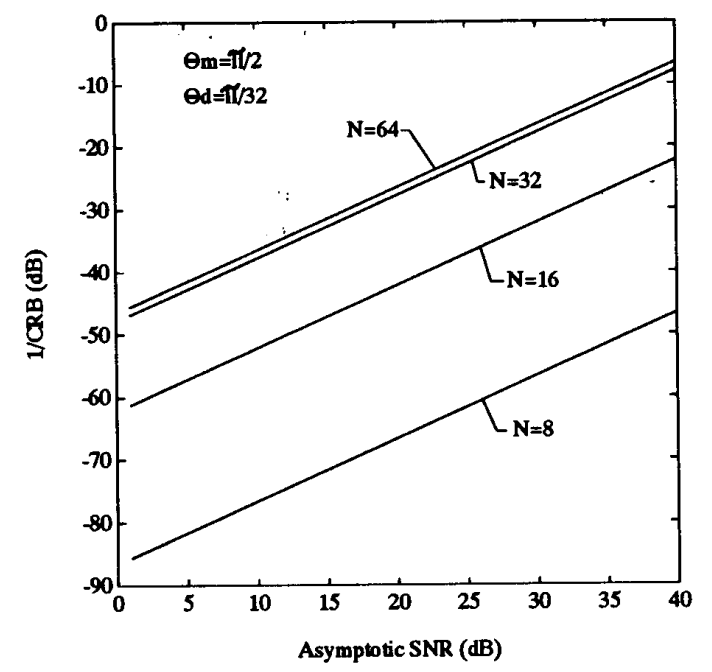

(b)

Fig. 12. CR Bounds for estimating $S\left(e^{j\left(\theta_{m}-\theta_{d}\right)}\right)$ (a) versus $N$ for several SNR and (b) versus SNR for several $N$.

\section{Conclusions}

Deterministic modal analysis is concerned with the identification of ARMA parameters, mode parameters, and energy spectra for deterministic modal signals observed in noise over a finite interval of time. In this paper we have presented a comprehensive study of performance bounds for these problems. Our results have been constructed from a simple characterization of the Fisher matrix for ARMA parameters together with sensitivity matrices that permit one to go back and forth between alternative parametric descriptions of a mode. Two such descriptions are the modal description and the rational energy spectrum for the mode. We have supplemented analytical findings with numerical results. The numerical results have been presented largely as confidence intervals that characterize best achievable performance when estimating pairs of parameters, such as amplitude and phase or damping coefficient and frequency. Our results for mode resolution from parameter estimates or from spectrum estimates illustrate again that the Rayleigh limit to resolution is difficult to beat, except at very high SNRs. The Rayleigh limit manifests itself as a threshold effect in the CR bound when that bound is plotted versus sample size. The results of this paper are independent of which technique-linear prediction, subspace identification, maximum likelihood, etc. - is used. They extend readily to deterministic modal analysis for direction-of-arrival estimation in narrow-band, equispaced, linear arrays, and to deterministic modal analysis for simultaneous frequency estimation and direction-of-arrival estimation.

\section{REFERENCES}

[1] G. R. de Prony and R. Baron, "Essai experimental et analytique: Sur les lois de la dilatabilité de fluides elastiques et sur celle de la force expansive de la vapeur de l'eau et de la vapeur de l'alcool, à différentes températures," J. l'Ecole Polytechnique, Paris, vol. 1, no. 2, pp. 24-76, 1795.

[2] D. Tufts and R. Kumaresan, "Frequency estimation of multiple sinusoids: Making linear prediction work like maximum likelihood," Proc. IEEE, vol. 70, pp. 975-990, Mar. 1983.

[3] R. Kumaresan and D. W. Tufts, "Estimating the parameters of exponentially damped sinusoids and pole-zero modeling in noise," IEEE Trans. Acoust., Speech, Signal Processing, vol. ASSP-30, no. 6, pp. 833-840, Dec. 1982.

[4] R. Kumaresan, D. W. Tufts, and L. L. Scharf, "A Prony method for noisy data: Choosing the signal components and selecting the order in exponential signal models,' Proc. IEEE, vol. 72, no. 2, pp. 230233, Feb. 1984

[5] A. G. Evans and R. Fischl, "Optimal least squares time-domain synthesis of recursive digital filters," IEEE Trans. Audio Electroacoustics, vol. AE-21, pp. 61-65, Feb. 1973.

[6] R. Kumaresan, L. L. Scharf, and A. K. Shaw, "An algorithm for pole-zero modelling and spectral analysis," IEEE Trans. Acoust., Speech, Signal Processing, vol. ASSP-34, no. 3, pp. 637-640, June 1986.

[7] Y. Bresler and A. Macovski, "Exact maximum likelihood parameter estimation of superimposed exponential signals in noise," IEEE Trans. Acoust., Speech, Signal Processing, vol. ASSP-34, no. 5, pp. 1081-1089, Oct. 1986.

[8] G. Bienvenu and L. Kopp, "Principe de la goniométrie passive adaptive," in Proc. VII EME Colloque GRETSI, Nice, France, 1979, pp. 106.1-106.10.

[9] R. O. Schmidt, "A signal subspace approach to multiple emitter location and spectral estimation," Ph.D. dissertation, Stanford University, 1981 .

[10] S. Y. Kung, "A new identification and model reduction algorithm via singular value decomposition," in Proc. 12th Asilomar Conf. Circuits, Syst., Comput., Pacific Grove, CA, Nov. 1978.

[11] R. Roy and T. Kailath, ESPRIT-Estimation of Signal Parameters via Rotational Invariance Techniques in SVD and Signal Processing, E. F. Deprettere, Ed. Amsterdam: North-Holland, 1988, pp. 233-265.

[12] D. W. Tufts, F. Giannella, I. Kirsteins, and L. L. Scharf, "CramerRao bounds on the accuracy of autoregressive parameter estimators," in Proc. IEEE Second Workshop Spectrum Anal., Tampa, FL, Nov. 1983.

[13] P. Stoica and A. Nehorai, "MUSIC, maximum likelihood, and Cramer-Rao bound," IEEE Trans. Acoust., Speech, Signal Processing, vol. 37, no. 5, pp. 720-741, May 1989.

[14] L. L. Scharf, Statistical Signal Processing. Reading, MA: AddisonWesley, 1991, ch. 6 .

[15] J.-P. Dugré, A. A. Beex, and L. L. Scharf, "Generating covariance sequences and the calculation of quantization and rounding error variances in digital filters," IEEE Trans. Acoust., Speech, Signal Processing, vol. ASSP-28, pp. 102-104, Feb. 1980. 
[16] C. Demeure and C. T. Mullis, "The Euclid algorithm and the fast computation of cross-covariance and autocovariance sequences," IEEE Trans. Acoust., Speech, Signal Processing, vol. 37, no. 4, pp. 545-552, Apr. 1989.

[17] C. Mullis, Course Notes, ECEN 7632, University of Colorado, Spring 1991.

[18] L. L. Scharf and L. T. McWhorter, "Geometry of the Cramer-Rao bound," Signal Processing, submitted for publication.

[19] B. Friedlander and B. Porat, "A general lower bound for parametric spectrum estimation," IEEE Trans. Acoust., Speech, Signal Processing, vol. ASSP-32, no. 4, pp. 728-733, Aug. 1984.

[20] B. Friedlander, "On the computation of the Cramer-Rao bound for ARMA parameter estimation," IEEE Trans. Acoust., Speech, Signal Processing, vol. ASSP-32, no. 4, pp. 721-727, Aug. 1984.

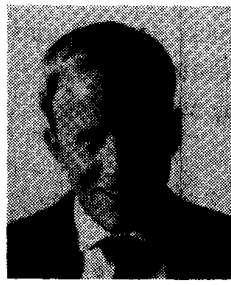

Todd McWhorter received the B.S. degree in electrical engineering from Colorado State University in 1984, and the M.S. degree in electrical engineering from the University of Colorado in 1991. He is currently pursuing the Ph.D. degree in electrical engineering at the University of Colorado.

From 1985 to 1989 he was employed by Wood ward Governor Company, Fort Collins, $\mathrm{CO}$, as an Applications Engineer and Circuit Designer. His research interests include modal analysis and array processing algorithms, Cramer-Rao bound theory, and spectral estimation.

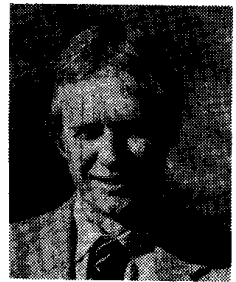

Louis L. Scharf (S'67-M'69-SM'77-F'86) re ceived the Ph.D. degree in electrical engineering from the University of Washington, Seattle, in 1969.

From 1969 to 1971 , he was a member of the Technical Staff at Honeywell's Marine Systems Center in Seattle. He was a faculty member at Colorado State University, Fort Collins, from 1971 to 1981, where he last served as a Professor of Electrical Engineering and Statistics. From 1982 to 1985 , he was a Professor and the Chairman of Electrical Engineering at the University of Rhode Island, Kingston. $\mathrm{He}$ is currently a Professor of Electrical and Computer Engineering at the University of Colorado, Boulder, where he teaches and conducts research in signal processing. In 1974 he was a Visiting Associate Professor at Duke University, Durham, NC. In 1977 he was at the University of South Paris, Orsay, where he was a member of the Technical Staff in the CNRS Laboratoire des Signaux et Systemes, Gif-sur-Yvette. In 1981 he was a Visiting Professor at the Ecole Nationale Supérieure des Télécommunications, Paris. In 1992 he was a Visiting Professor at Institut Eurécom, Sophia-Antipolis, France. He has served as a consultant to Hewlett-Packard, Honeywell Inc., the Applied Physics Labs Seattle, the Research Triangle Institute, Green Mountain Geophysics, and Ball Aerospace Corporation.

Dr. Scharf is a past member of the ASSP ADCOM and the Editorial Board of Signal Processing. He is a past Associate Editor for the IEEE Transactions on Acoustics, Speech, and Signal Processing. He was the Technical Program Chairman for the 1980 International Conference on ASSP. He is a member of Eta Kappa Nu. 